\title{
Title: A conserved regulatory program drives emergence of the lateral plate mesoderm
}

Authors: Christopher Hess ${ }^{1, \uparrow}$, Karin D. Prummel ${ }^{1, \uparrow}$, Susan Nieuwenhuize ${ }^{1}$, Hugo J. Parker ${ }^{2,3}$, Katherine W. Rogers ${ }^{4}$, Iryna Kozmikova $^{5}$, Claudia Racioppi ${ }^{6}$, Sibylle Burger ${ }^{1}$, Eline C. Brombacher ${ }^{1}$, Alexa Burger ${ }^{1}$, Anastasia Felker ${ }^{1}$, Elena Chiavacci ${ }^{1}$, Gopi Shah $^{7}$, Jan Huisken ${ }^{7,8}$, Zbynek Kozmik ${ }^{5}$, Lionel Christiaen ${ }^{6}$, Patrick Müller ${ }^{4}$, Marianne Bronner ${ }^{2}$, Robb Krumlauf ${ }^{3}$, Christian Mosimann ${ }^{1, *}$.

\author{
Affiliations: \\ ${ }^{1}$ Institute of Molecular Life Sciences, University of Zürich, Zürich, Switzerland \\ ${ }^{2}$ California Institute of Technology, Pasadena, United States \\ ${ }^{3}$ Stowers Institute for Medical Research, Kansas City, United States \\ ${ }^{4}$ Friedrich-Miescher-Laboratory of the Max Planck Society, Tübingen, Germany \\ ${ }^{5}$ Institute of Molecular Genetics of the ASCR, Prague, Czech Republic \\ ${ }^{6}$ New York University College of Arts and Science, New York, United States \\ ${ }^{7}$ Max Planck Institute of Molecular Cell Biology and Genetics, Dresden, Germany \\ ${ }^{8}$ Morgridge Institute for Research, Madison, Wisconsin, United States \\ ${ }^{\dagger}$ contributed equally to this work \\ * correspondence to: christian.mosimann@imls.uzh.ch
}

\section{Abstract:}

Cardiovascular cell lineages emerge with kidney, smooth muscle, and limb skeleton progenitors from the lateral plate mesoderm (LPM). How the LPM emerges during development and how it has evolved to form key lineages of the vertebrate body plan remain unknown. Here, we captured LPM formation by transgenic in toto imaging and lineage tracing using the first pan-LPM enhancer element from the zebrafish gene draculin $(d r l)$. $d r l$ LPM enhancer-based reporters are specifically active in LPM-corresponding territories of several chordate species, uncovering a universal LPM-specific gene program. Distinct from other mesoderm, we identified EomesA, FoxH1, and MixL1 with BMP/Nodal-controlled Smad activity as minimally required factors to drive $d r l$-marked LPM formation. Altogether, our work provides a developmental and mechanistic framework for LPM emergence and the in vitro differentiation of cardiovascular cell types. Our findings suggest that the LPM may represent an ancient cell fate domain that predates ancestral vertebrates. 


\section{Main Text:}

Following gastrulation, mesoderm in vertebrates is classically described to partition into axial, paraxial, and lateral domains(Gurdon, 1995). The ventrally forming lateral plate mesoderm (LPM) is composed of highly mobile cells and is mainly defined by its position adjacent to the somite-forming paraxial mesoderm (Hatada and Stern, 1994). Transplantation and lineage tracing experiments in several species have shown that the LPM contains progenitor cells of the circulatory system, smooth muscle lineages, the kidneys (often demarcated as dedicated intermediate mesoderm), and the limb anlagen (Chal and Pourquié, 2017; Gurdon, 1995; Takasato and Little, 2015). Several transcription factors including Hand1/2, Tbx5, Osr1, FoxF1, Prrx1, Mesp1, and Etv2 are expressed in LPM territories and play overlapping roles in cell fate determination (Chal and Pourquié, 2017; Davidson and Zon, 2004; Takasato and Little, 2015), albeit not always with conserved function (Yabe et al., 2016). Anterior-to-posterior expression domains of select transcription factors including Tbx1/10 and Hand are conserved in lampreys and even in the basal cephalochordate amphioxus that lacks major LPM-derived organ systems (Onimaru et al., 2011). These observations raise the possibility of the existence of an ancient upstream program that delineates the prospective LPM domain.

In zebrafish, the LPM emerges from the ventral half of the embryo and becomes readily detectable as a patchwork of bilateral gene expression domains at the end of gastrulation (Davidson and Zon, 2004). hand2 expression marks in the anterior LPM (ALPM) the heart field and pectoral fin precursors, and a lateral-most domain within the posterior LPM (PLPM) (Perens et al., 2016; Yelon et al., 2000), while pax2.1 outlines the prospective kidney progenitors that in zebrafish become positioned more laterally than in chick and mouse (Perens et al., 2016; Takasato and Little, 2015). Hematopoietic and endothelial progenitors in distinct ALPM and PLPM domains express a number of lineage-determining transcription factor genes including etv2, scl/tall, lmo2, and later gatal, pu.1, and flil (Davidson and Zon, 2004). While several studies have uncovered mechanisms that control the lateral-to-medial or anteriorposterior specification of LPM-descendant organ precursors (Chal and Pourquié, 2017; Helker et al., 2015; Tonegawa et al., 1997), how the LPM arises among other mesendoderm remains incompletely understood.

Several cis-regulatory elements have been reported with specific activity in the mouse LPM; these include a small upstream enhancer of mouse and human HoxB6 (Becker et al., 1996), an upstream enhancer of mouse Gata4 (Rojas et al., 2005), and a downstream enhancer of mouse Bmp4 (Chandler et al., 2009). In line with the ventral origin of the LPM, the Gata4 LPM enhancer responds to Smads downstream of BMP signaling (Rojas et al., 2005). Nonetheless, the activities driven by these enhancer elements in mice are confined to the PLPM. In contrast, a transgenic reporter from the $6.35 \mathrm{~kb}$ cis-regulatory region of the zebrafish-specific gene draculin ( $d r l)$ specifically outlines seemingly all LPM derivatives and labels the forming and differentiating LPM from its 
emergence in late gastrulation (Mosimann et al., 2015), as confirmed by Cre/lox-mediated genetic lineage analysis (Felker et al., 2016; Gays et al., 2017; Henninger et al., 2016; Mosimann et al., 2015). These observations suggest that $-6.35 d r l$ harbors one or several cis-regulatory elements active throughout the prospective LPM from gastrulation onwards, raising the intriguing possibility that it may provide a read-out of an LPM-specific program.

To resolve the dynamics of LPM emergence marked by the $-6.35 \mathrm{drl}$ cis-regulatory elements, we performed 3D single-plane illumination microscopy (SPIM) (Fig. 1A-D) and panoramic projections (Schmid et al., 2013) (Fig. 1E-H) of -6.35drl:EGFP reporter transgenic zebrafish embryos. -6.35drl:EGFP-expressing LPM precursors became detectable by early gastrula stages (50\% epiboly) and slowly condensed along the embryo margin through the end of gastrulation (tailbud stage) (Fig. 1A,B,F; Movie S1, S2). From tailbud stage onward, -6.35drl:EGFP-marked LPM formed a continuous band of cells with condensing anterior and posterior segments interspersed by a scattered medial segment (Fig. 1C,D,G,H). We demonstrated that this EGFP-positive cell band encompasses stripes of previously characterized bilateral LPM sub-domain markers (Fig. 1I-L): lmo2:dsRED2 labels embryonic hematopoietic and vascular tissues (Zhu et al., 2005), and its expression overlapped with medial -6.35drl:EGFP-expressing cells in the ALPM and PLPM (Fig. 1L); scl:EGFP (Zhang and Rodaway, 2007) also co-expressed with -6.35drl:mCherry in the most medial PLPM domain and in a small ALPM population (Fig. 1J); pax2.1:EGFP in early somitogenesis marks pronephric epithelial precursors in zebrafish (Picker et al., 2002), and its expression overlaps with -6.35drl:mCherry in the PLPM, while the pax2.1:EGFPexpressing midbrain-hindbrain precursor cells of ectodermal origin were negative for $-6.35 \mathrm{drl}$ :mCherry (Fig. 1K). Moreover, hand2:EGFP expression, which demarcates the lateral-most PLPM domain and parts of the ALPM-derived heart field and pectoral fin precursors (Perens et al., 2016; Yelon et al., 2000), is also fully situated within the pan-LPM expression domain of 6.35drl:mCherry (Fig. 1L). Taken together, these data reveal that transgenic reporters based on $-6.35 d r l$ demarcate the entire emerging zebrafish LPM (Fig. 1M).

To clarify how the $d r l$-expressing LPM emerges relative to the endoderm during gastrulation, we performed time lapse imaging of the forming LPM in toto. Panoramic SPIM for the sox17:EGFP-positive endoderm together with -6.35drl:mCherry reporter expression revealed sox 17; $d r l$ reporter double-positive cells from the onset of reporter detection through late gastrulation (Fig. 1NO; Fig. S1A-D). After gastrulation, we detected a continuous band of $-6.35 \mathrm{drl}$-positive cells around the developing embryo that was clearly separated from the more medial endodermal sox17 expression domain (Fig. 1N; Fig. S1D; Movie S3, S4). The temporal overlap in expression suggests that the $-6.35 \mathrm{drl}$-marked territory initially emerges among mesendoderm. Consequently, we performed -6.35drl:creERT2-based genetic lineage tracing with hsp70l:switch (hsp70l:loxP-STOP-loxP-EGFP) with 4-OHT- 
induction at shield and 5-6 somite stages (ss) to explore whether endoderm derivatives are marked by the $-6.35 \mathrm{drl}$ reporter at $72 \mathrm{hpf}$ (Fig. 1P). 4-OHT induction at shield stage (Fig. 1Q,T) broadly marked i) LPM lineages as the only mesodermal fates (Fig. 1Q), and ii) broad territories within endodermal organs including pancreas, liver, and pharynx/gut epithelium (Fig. 1Q-T; Fig. S2B-E). In contrast, sox17:creERT2 exclusively marked endoderm lineages at all induction time points tested (Fig. S2I-K), in line with sox17 expression demarcating specified endoderm downstream of the key zebrafish endoderm regulator sox32 (Alexander and Stainier, 1999). Supporting our SPIM observations of the $-6.35 \mathrm{drl}$ reporter and consistent with previous studies (Felker et al., 2016; Gays et al., 2017; Henninger et al., 2016; Mosimann et al., 2015), -6.35drl:creERT2-based lineage tracing with 4-OHT induction at 5-6 ss efficiently mapped to LPM lineages with only minimal endoderm contribution (Fig. 1U; Fig. S2B,E). These results are not caused by lineage-biased loxP reporter sensitivity, as controlled for by recombining hsp70l:switch with the ubiquitous ubi:creERT2 (Fig. S2B,F-H). Embryos that fail to form endoderm upon perturbation of sox32 (Alexander and Stainier, 1999) still properly generated 6.35drl-traced LPM that patterns into its major descendants including heart, blood, endothelium, and pronephros (Fig. S3). These data establish that during gastrulation, $-6.35 \mathrm{drl}$-marked LPM gradually refines from a ventral mesendoderm territory to a bilateral LPM domain along the entire anterior-posterior axis of the embryo. The concomitant $d r l$;sox 17 -expressing progenitors contribute to seemingly all endodermal organs. The rather rare lineage labeling of somitic muscle by -6.35drl:creERT2 (Fig. 1Q) (Gays et al., 2017; Mosimann et al., 2015) further suggests that, in zebrafish, the paraxial mesoderm and the LPM develop as distinct mesoderm lineages with minimal overlap (Warga and Nüsslein-Volhard, 1999).

To identify the cis-regulatory components in the $-6.35 \mathrm{drl}$ genomic region responsible for pan-LPM expression, we sub-divided $6.35 d r l$ into smaller regions and tested them in reporter assays (Fig. 2A). These efforts uncovered $d r l$ cis-regulatory elements surrounding exon 1 that recapitulated strongest $d r l$ expression during somitogenesis in the differentiating cardiovascular/hematopoietic ALPM and PLPM $\left(d r l^{\text {proximal }}\right)$ and selectively in the PLPM $\left(d r l^{P L P M}\right)$, respectively (Fig. 2B,C). In addition, we found a 968 bp region in the distal first intron, referred to as $d r l^{L P M}$, that as transgenic reporter fully recapitulated early $-6.35 \mathrm{drl}$ and endogenous $d r l$ expression in zebrafish embryos before fading during somitogenesis (approx. $13 \mathrm{hpf}$ ) (Fig. 2D). Corresponding to gastrulation-stage lineage tracing with -6.35drl:creERT2 (Fig. 1P-U), genetic lineage tracing with $d r{ }^{L P M}: c r e E R T 2$ and hsp70l:switch (Fig. 2E) induced at 50\% epiboly specifically labels LPM-derived organs including heart, iSMCs, blood, endothelium, and kidney, and additionally marked endodermal cells (Fig. 2F-H). Trimming of $d r l^{L P M}$ resulted a minimal enhancer region of $432 \mathrm{bp}\left(d r l^{\min L P M}\right)$ that still behaved as a pan-LPM enhancer and lost its specificity and activity upon further trimming (Fig. S4). Hence, the $-6.35 \mathrm{drl}$ reporter pattern combines an early mesendoderm/pan-LPM phase and a later ALPM/PLPM-restricted phase 
controlled by dedicated cis-regulatory elements, consistent with $d r l$-based lineage tracing (Fig. 1P-U) (Felker et al., 2016; Gays et al., 2017; Henninger et al., 2016; Mosimann et al., 2015).

We next investigated whether the $d r l^{L P M}$ enhancer reads out a conserved pan-LPM program by performing reporter assays in other chordate species. Electroporation of $d r l^{L P M}$ reporter into the primitive streak of ex ovo-cultured chicken embryos at $\mathrm{HH} 3+/ 4$ resulted in specific reporter activity in the forming LPM. Depending on the stage and region of electroporation, we observed specific reporter activity in medial LPM stripes or the posterior end of the LPM, including outward migrating cells reminiscent of endothelial/blood progenitors at later stages (Fig. 3A; Fig. S5B-J). In contrast, the previously characterized posterior LPM enhancers of mouse Gata4 (Rojas et al., 2005) and mouse Bmp4a (Chandler et al., 2009) showed specific activity only in the outward migrating endothelial/blood progenitors in chicken embryos (Fig. S6A,B), while the LPM enhancer of mouse HoxB6 (Becker et al., 1996) showed no specific activity (Fig. S6c). When injected in zebrafish embryos, these constructs showed expression mainly in the notochord and lacked specific LPM activity (Fig. S6D-G). Furthermore, there are species-specific differences in endogenous genes, as FoxF1 in mice is expressed in the early developing LPM (Mahlapuu et al., 2001), while zebrafish foxf1 mRNA is not detectable by in situ hybridization (ISH) before late somitogenesis (Fig. S6H-K). Taken together, these results indicate that activities of previously used LPM reporters do not universally extrapolate to other vertebrates, but that the $d r l^{L P M}$ enhancer does react to an LPMspecific program in zebrafish and chicken.

We next explored the ability of the $d r l^{L P M}$ enhancer to read out a pan-LPM program in distinct chordate species. Lampreys are jawless fish that can provide unique insights into vertebrate evolution due to their position at the base of the vertebrate phylogeny. Microinjection of $d r l^{L P M}$ and $d r l^{m i n L P M}$ EGFP-based reporters into sea lamprey embryos (Petromyzon marinus) consistently resulted in strong EGFP expression in the mesendoderm starting during neurulation (stage (st) 18) ( $\mathrm{n}=145 / 231$ ), as well as in the developing pharynx at st 22-24 (Fig. 3B-F). Transverse embryo sections revealed that this early expression domain partially includes the anteriormost expression of lamprey pmHandA that expresses in LPM (Onimaru et al., 2011), with the later pharyngeal expression of EGFP being restricted to the endoderm and mesoderm (Fig. S7A-I).

Electroporation of $d r l^{L P M}$ reporters into embryos of the tunicate Ciona robusta, a chordate species belonging to a sister clade of the vertebrates, revealed specific reporter activity in emerging cardiac and pharyngeal muscle lineages at larval stage (st 26; 18 hpf) (Fig. 3G) (Hotta et al., 2007). We observed $d r l^{L P M_{-}}$and $d r l^{\min L P M_{-}}$driven EGFP reporter activity specifically in the atrial siphon muscle precursors (ASMPs) and in both first and second heart precursors (FHPs and SHPs) as confirmed by co-localization of Mesp:H2B$m$ Cherry expression that labels the cardiopharyngeal cell lineage ( $\mathrm{n}=15 / 92$ and 23/110 respectively; Fig. S7J-L). We rarely observed 
EGFP expression in the anterior tail muscles (ATMs) of the Ciona larval tail, suggesting that $d r l$ reporters drive expression specifically in the emerging multipotent cardiopharyngeal progenitors. Moreover, using both $d r l$ reporters, we observed ectopic reporter activity in the mesenchyme, which often activates transgenes non-specifically (Stolfi and Christiaen, 2012).

Lastly, in the cephalochordate amphioxus (Branchiostoma lanceolatum), which belongs to the most basally divergent lineage of chordates (Bertrand et al., 2011), ventral mesoderm that is considered to be equivalent to the vertebrate LPM originates from the ventral half of the somite territory. At mid-neurula stage, the ventral wall of the somite is evaginating as nascent ventral mesoderm, which by late neurula stage fuses at the midline under the gut endoderm (Holland and Holland, 2007; Kozmikova and Kozmik, 2015). Indeed, amphioxus Hand, Csx, Vent1, and AmphiNk2-tin are expressed in the ventral half of the somite territory at mid-neurula stage (Holland and Holland, 2007; Kozmikova and Kozmik, 2015; Onimaru et al., 2011). Correspondingly, our injection-based reporter assays in amphioxus showed $d r l^{L P M}$ reporter activity at mid-neurula stage $(6 / 7 \mathrm{ss})$ in the ventral half of somites and evaginating somites $(n=61 / 65)$ (Fig. 3H, Fig. S7M,N).

Taken together, these observations establish that the $d r l^{L P M}$ regulatory module in the zebrafish $d r l$ locus is capable of reading out an evolutionarily conserved pan-LPM program that is active from chordates to higher vertebrates.

We next sought to gain insight into what upstream input controls the $d r l^{L P M}$ enhancer. Mesendoderm induction is coordinated by marginal Nodal and ventral BMP signaling by controlling a range of downstream target genes. In line with ventral LPM emergence, endogenous $d r l$ expression was virtually absent in embryos maternally mutant for dominant Smad5 ( $s b n)$ that are devoid of ventral BMP activity (Hild et al., 1999) (Fig. 4A,B). In contrast, MZoep-mutant embryos lost the dorsal $d r l$ expression domain but still weakly expressed $d r l$ throughout the marginal zone, despite their inability to relay Nodal signaling in the gastrulating embryo (Fig. 4C). Consistent with our lineage tracing results (Fig. S3), embryos devoid of endoderm upon sox32 knockdown also still expressed $d r l$ (Fig. 4D). These results underscore the importance of ventral BMP and marginal Nodal signaling in modulating early $d r l$ expression.

To uncover transcriptionally responsive regions within the $d r l^{L P M}$ enhancer, we targeted $d r l^{L P M}$ with a small library of sgRNAs to introduce maximally efficient Cas9-mediated deletions in zebrafish embryos transgenic for -6.35drl:EGFP or $d r l^{L P M}: E G F P$ (Fig. S8A) (Burger et al., 2016; Lindsay et al., 2016). This analysis revealed two mutagenesis-sensitive regions that caused mosaic loss of $d r l^{L P M}$ and $-6.35 d r l$ reporter activity at mid-gastrulation/bud stage (Fig. S8B-E); our mutagenesis of the early-acting $d r l^{L P M} \operatorname{did}$ not 
significantly perturb overall transgene functionality, as EGFP reporter activity driven by the later-acting regulatory elements in $6.35 d r l$ remained intact (Fig. S8B“,C”).

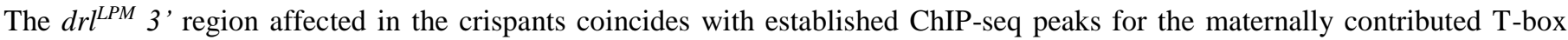
transcription factor Eomesodermin (Eomes) and its interaction partner FoxH1 that act with Nodal/BMP-mediating Smads; these factors together influence endodermal and mesodermal target genes (Charney et al., 2017; Nelson et al., 2014; Nelson et al., 2017; Slagle et al., 2011) including endogenous $d r l$ expression (Slagle et al., 2011) and bind the $d r l^{L P M}$ region (Fig. 4E) (Dubrulle et al., 2015; Nelson et al., 2014; Nelson et al., 2017). Ubiquitous expression of constitutively-active EomesA-VP16 strongly augmented and prolonged $d r l^{L P M}$ reporter expression in the native LPM domain up to mid-/late-somitogenesis compared to uninjected controls (Fig. 4F,G). Similarly, individual ubiquitous expression of native eomesA or foxhl mRNA strongly increased $d r l^{L P M}$ reporter expression within its native expression domain during mid-gastrulation (Fig. 4H-J).

From these observations, and since maternal eomesA and foxhl transcripts are seemingly ubiquitous during gastrulation (Bruce et al., 2003; Slagle et al., 2011), we reasoned that BMP/Nodal-based position information must have additional input on $d r l^{L P M}$ enhancer activity. To find this additional input, we performed individual and combinatorial mRNA-mediated misexpression of eomesA and foxhl with a variety of transcription factors implicated in early mesendoderm development (Fig. S9). These experiments identified the homeodomain transcription factor MixL1 as a third factor to act together with Eomes and FoxH1 in controlling $d r l^{L P M}$ enhancer activity (Fig. S9). MixL1 is a downstream target of BMP and Nodal signaling implicated in controlling endoderm and mesoderm fates (Kunwar et al., 2003; Stainier et al., 1996), retains ventral expression in MZoep mutants (Slagle et al., 2011), and triggers extensive blood formation at the expense of dorsal fates upon mRNA injection in Xenopus (Mead et al., 1996). Further, MixL1 can physically interact with Eomes (Bjornson et al., 2005) and MixL1, EomesA, FoxH1, and Smads have been detected by ChIP-Seq at the intronic $d r l^{L P M}$ enhancer region (Dubrulle et al., 2015; Nelson et al., 2017) (Fig. 4e). Mutating mixll by Cas9 resulted in mosaic loss of $d r l^{L P M}$ reporter activity in F0 crispants (Fig. S9). Mutating the redundant mixll paralog mezzo (Poulain and Lepage, 2002) alone or together with mixll did not influence $d r l^{L P M}$ reporter activity (Fig. S9), suggesting that predominantly MixL1 is acting on $d r l^{L P M}$.

Akin to eomesA and foxhl, native mixll mRNA alone increased $d r l^{L P M}$ reporter expression in the native LPM domain (Fig. 4K). In contrast, combining the triplet of eomesA, foxH1, and mixL1 (E/F/M) expressed from native ORF-encoding mRNA or from Tol2based DNA vectors led to ubiquitous $d r^{L P M}$ reporter activation (Fig. $4 \mathrm{~L}, \mathrm{M}$ ). Combining native $E / F / M$ in endoderm-perturbed 
embryos also resulted in ubiquitous $d r l^{L P M}$ reporter activation (Fig. $4 \mathrm{~N}$ ), suggesting that $d r l^{L P M}$ reporter-induced cells have an LPM identity. Similarly, we found $E / F / M$-triggered induction of tmem 88 a, expression of which is highly enriched in the early native LPM (Mosimann et al., 2015) (Fig. S10). In contrast, the expression domains of various mesodermal genes remained broadly unaffected (Fig. S10), as for hand2 expression that initiates in the lateral-most LPM only after gastrulation (Fig. S10; Fig. 1L). Even in sbnmutant embryos without ventral BMP signaling, the constitutive-active mRNA or Tol2-based DNA vector combination of $E / F / M$ induced $d r l$ expression dorsally, an activity that persisted even upon additional endoderm disruption (Fig. 4O; Fig. S11A,B). These observations suggest that $E / F / M$ overexpression requires additional Smad activity, which in $s b n$ is available in the dorsal Nodalreceiving domain. Conversely, loss of Nodal signaling in MZoep mutants led to a ventral upregulation of endogenous $d r l$ expression upon $E / F / M$ overexpression (Fig. S11C,D). Together, these data demonstrate that in zebrafish the combination of EomesA, FoxH1, and MixL1 potentiates ventral Smad-relayed signals to demarcate a mesendoderm territory to become prospective LPM, as read out by the $d r^{L P M}$ enhancer.

Its dynamic structure and the lack of a means to genetically mark the ventrally forming LPM in a specific manner has hindered concise comparative studies and in toto charting of the emergence and morphogenesis of the LPM. Here, we have shown that the $d r l^{L P M}$ enhancer reads out LPM-demarcating transcriptional activity in several chordate species, ranging from cephalochordates to amniotes, suggesting that the underlying positional program is of ancient evolutionary origin. In zebrafish, our data reveals that the transcription factors Eomes, FoxH1, and MixL1 are sufficient to trigger a basic LPM program. These factors have been previously implicated in mesendoderm development in various contexts, including blood formation (Mead et al., 1996); our observations now imply that, among their roles in mesendoderm regulation, they cooperate in inducing LPM together with ventral BMP-controlled and marginal Nodal-controlled Smad activity. These findings provide a developmental regulatory framework for the contribution of Eomes and MixL1 in early steps during programming naïve pluripotent stem cells into cardiac lineages (Pereira et al., 2012; Rao et al., 2015), suggesting that these factors in concert with the correct dose of BMP and Nodal establish the correct mesendodermal precursor lineage for forming cardiovascular-competent LPM.

\section{References}

Alexander, J. and Stainier, D. Y. (1999). A molecular pathway leading to endoderm formation in zebrafish. Curr. Biol. 9, 114757.

Becker, D., Eid, R. and Schughart, K. (1996). The limb/LPM enhancer of the murine Hoxb6 gene: reporter gene analysis in 
transgenic embryos and studies of DNA-protein interactions. Pharm. Acta Helv. 71, 29-35.

Bertrand, S., Escriva, H., Williams, N. A., Holland, N. D. and Holland, L. Z. (2011). Evolutionary crossroads in developmental biology: amphioxus. Development 138, 4819-30.

Bjornson, C. R. R., Griffin, K. J. P., Farr, G. H., Terashima, A., Himeda, C., Kikuchi, Y. and Kimelman, D. (2005). Eomesodermin Is a Localized Maternal Determinant Required for Endoderm Induction in Zebrafish. Dev. Cell 9, 523-533.

Bruce, A. E. E., Howley, C., Zhou, Y., Vickers, S. L., Silver, L. M., King, M. Lou and Ho, R. K. (2003). The maternally expressed zebrafish T-box gene eomesodermin regulates organizer formation. Development 130, 5503-5517.

Burger, A., Lindsay, H., Felker, A., Hess, C., Anders, C., Chiavacci, E., Zaugg, J., Weber, L. M. L. M., Catena, R., Jinek, M., et al. (2016). Maximizing mutagenesis with solubilized CRISPR-Cas9 ribonucleoprotein complexes. Development 143, $2025-37$.

Chal, J. and Pourquié, O. (2017). Making muscle: skeletal myogenesis in vivo and in vitro. Development 144,.

Chandler, K. J., Chandler, R. L. and Mortlock, D. P. (2009). Identification of an ancient Bmp4 mesoderm enhancer located 46 kb from the promoter. Dev. Biol. 327, 590-602.

Charney, R. M., Forouzmand, E., Cho, J. S., Cheung, J., Paraiso, K. D., Yasuoka, Y., Takahashi, S., Taira, M., Blitz, I. L., Xie, X., et al. (2017). Foxh1 Occupies cis -Regulatory Modules Prior to Dynamic Transcription Factor Interactions Controlling the Mesendoderm Gene Program. Dev. Cell 40, 595-607.e4.

Davidson, A. J. and Zon, L. I. (2004). The "definitive" (and "primitive") guide to zebrafish hematopoiesis. Oncogene 23, 72337246.

Dubrulle, J., Jordan, B. M., Akhmetova, L., Farrell, J. A., Kim, S.-H., Solnica-Krezel, L. and Schier, A. F. (2015). Response to Nodal morphogen gradient is determined by the kinetics of target gene induction. Elife 4,.

Felker, A., Nieuwenhuize, S., Dolbois, A., Blazkova, K., Hess, C., Low, L. W. L. W. L., Burger, S., Samson, N., Carney, T. J. T. J., Bartunek, P., et al. (2016). In Vivo Performance and Properties of Tamoxifen Metabolites for CreERT2 Control. PLoS One 11, e0152989.

Gays, D., Hess, C., Camporeale, A., Ala, U., Provero, P., Mosimann, C. and Santoro, M. M. (2017). An exclusive cellular and molecular network governs intestinal smooth muscle cell differentiation in vertebrates. Development 144, dev.133926.

Gurdon, J. B. (1995). The Formation of Mesoderm and Muscle in Xenopus. In Organization of the Early Vertebrate Embryo, pp. 51-59. Boston, MA: Springer US.

Hatada, Y. and Stern, C. (1994). A fate map of the epiblast of the early chick embryo. Development 120, $2879-2889$.

Helker, C. S. M., Schuermann, A., Pollmann, C., Chng, S. C., Kiefer, F., Reversade, B. and Herzog, W. (2015). The 
hormonal peptide Elabela guides angioblasts to the midline during vasculogenesis. Elife 4, e06726.

Henninger, J., Santoso, B., Hans, S., Durand, E., Moore, J., Mosimann, C., Brand, M., Traver, D. and Zon, L. (2016). Clonal fate mapping quantifies the number of haematopoietic stem cells that arise during development. Nat. Cell Biol. 19, 17-27.

Hild, M., Dick, A., Rauch, G. J., Meier, A., Bouwmeester, T., Haffter, P. and Hammerschmidt, M. (1999). The smad5 mutation somitabun blocks Bmp2b signaling during early dorsoventral patterning of the zebrafish embryo. Development 126, 2149-59.

Holland, L. Z. and Holland, N. D. (2007). A revised fate map for amphioxus and the evolution of axial patterning in chordates. Integr. Comp. Biol. 47, 360-72.

Hotta, K., Mitsuhara, K., Takahashi, H., Inaba, K., Oka, K., Gojobori, T. and Ikeo, K. (2007). A web-based interactive developmental table for the ascidian Ciona intestinalis, including 3D real-image embryo reconstructions: I. From fertilized egg to hatching larva. Dev. Dyn. 236, 1790-1805.

Kozmikova, I. and Kozmik, Z. (2015). Gene regulation in amphioxus: An insight from transgenic studies in amphioxus and vertebrates. Mar. Genomics 24, 159-166.

Kunwar, P. S., Zimmerman, S., Bennett, J. T., Chen, Y., Whitman, M. and Schier, A. F. (2003). Mixer/Bon and FoxH1/Sur have overlapping and divergent roles in Nodal signaling and mesendoderm induction. Development 130, 5589-99.

Lindsay, H., Burger, A., Biyong, B., Felker, A., Hess, C., Zaugg, J., Chiavacci, E., Anders, C., Jinek, M., Mosimann, C., et al. (2016). CrispRVariants charts the mutation spectrum of genome engineering experiments. Nat. Biotechnol. 34, 701-702.

Mahlapuu, M., Ormestad, M., Enerbäck, S. and Carlsson, P. (2001). The forkhead transcription factor Foxf1 is required for differentiation of extra-embryonic and lateral plate mesoderm. Development 128, 155-66.

Mead, P. E., Brivanlou, I. H., Kelley, C. M. and Zon, L. I. (1996). BMP-4-responsive regulation of dorsal-ventral patterning by the homeobox protein Mix.1. Nature 382, 357-360.

Mosimann, C., Panáková, D., Werdich, A. A. A., Musso, G., Burger, A., Lawson, K. L. K. L., Carr, L. A. L. A., Nevis, K. R. K. R., Sabeh, M. K. K., Zhou, Y., et al. (2015). Chamber identity programs drive early functional partitioning of the heart. Nat. Commun. 6, 8146.

Nelson, A. C., Cutty, S. J., Niini, M., Stemple, D. L., Flicek, P., Houart, C., Bruce, A. and Wardle, F. C. (2014). Global identification of Smad2 and Eomesodermin targets in zebrafish identifies a conserved transcriptional network in mesendoderm and a novel role for Eomesodermin in repression of ectodermal gene expression. BMC Biol. 12, 81 .

Nelson, A. C., Cutty, S. J., Gasiunas, S. N., Deplae, I., Stemple, D. L. and Wardle, F. C. (2017). In Vivo Regulation of the Zebrafish Endoderm Progenitor Niche by T-Box Transcription Factors. Cell Rep. 19, 2782-2795. 
Onimaru, K., Shoguchi, E., Kuratani, S. and Tanaka, M. (2011). Development and evolution of the lateral plate mesoderm: comparative analysis of amphioxus and lamprey with implications for the acquisition of paired fins. Dev. Biol. 359, 124-36.

Pereira, L. A., Wong, M. S., Mossman, A. K., Sourris, K., Janes, M. E., Knezevic, K., Hirst, C. E., Lim, S. M., Pimanda, J. E., Stanley, E. G., et al. (2012). Pdgfr $\alpha$ and Flk1 are direct target genes of Mixl1 in differentiating embryonic stem cells. Stem Cell Res. 8, 165-179.

Perens, E. A., Garavito-Aguilar, Z. V, Guio-Vega, G. P., Peña, K. T., Schindler, Y. L. and Yelon, D. (2016). Hand2 inhibits kidney specification while promoting vein formation within the posterior mesoderm. Elife 5, e19941.

Picker, A., Scholpp, S., Böhli, H., Takeda, H. and Brand, M. (2002). A novel positive transcriptional feedback loop in midbrain-hindbrain boundary development is revealed through analysis of the zebrafish pax 2.1 promoter in transgenic lines. Development 129, 3227-39.

Poulain, M. and Lepage, T. (2002). Mezzo, a paired-like homeobox protein is an immediate target of Nodal signalling and regulates endoderm specification in zebrafish. Development $\mathbf{1 2 9}$,.

Rao, J., Pfeiffer, M. J., Frank, S., Adachi, K., Piccini, I., Quaranta, R., Araúzo-Bravo, M., Schwarz, J., Schade, D., Leidel, S., et al. (2015). Stepwise Clearance of Repressive Roadblocks Drives Cardiac Induction in Human ESCs. Cell Stem Cell.

Rojas, A., De Val, S., Heidt, A. B., Xu, S.-M., Bristow, J. and Black, B. L. (2005). Gata4 expression in lateral mesoderm is downstream of BMP4 and is activated directly by Forkhead and GATA transcription factors through a distal enhancer element. Development 132, 3405-17.

Schmid, B., Shah, G., Scherf, N., Weber, M., Thierbach, K., Campos, C. P., Roeder, I., Aanstad, P. and Huisken, J. (2013). High-speed panoramic light-sheet microscopy reveals global endodermal cell dynamics. Nat Commun 4, 2207.

Slagle, C. E., Aoki, T. and Burdine, R. D. (2011). Nodal-dependent mesendoderm specification requires the combinatorial activities of FoxH1 and Eomesodermin. PLoS Genet. 7, e1002072.

Stainier, D. Y., Fouquet, B., Chen, J. N., Warren, K. S., Weinstein, B. M., Meiler, S. E., Mohideen, M. A., Neuhauss, S. C., Solnica-Krezel, L., Schier, A. F., et al. (1996). Mutations affecting the formation and function of the cardiovascular system in the zebrafish embryo. Development 123, 285-92.

Stolfi, A. and Christiaen, L. (2012). Genetic and genomic toolbox of the chordate Ciona intestinalis. Genetics 192, 55-66.

Takasato, M. and Little, M. H. (2015). The origin of the mammalian kidney: implications for recreating the kidney in vitro. Development 142, 1937-1947.

Tonegawa, A., Funayama, N., Ueno, N. and Takahashi, Y. (1997). Mesodermal subdivision along the mediolateral axis in chicken controlled by different concentrations of BMP-4. Development 124,. 
Warga, R. M. and Nüsslein-Volhard, C. (1999). Origin and development of the zebrafish endoderm. Development 126, 827-38.

Yabe, T., Hoshijima, K., Yamamoto, T. and Takada, S. (2016). Quadruple zebrafish mutant reveals different roles of Mesp genes in somite segmentation between mouse and zebrafish. Development 143,.

Yelon, D., Ticho, B., Halpern, M. E., Ruvinsky, I., Ho, R. K., Silver, L. M. and Stainier, D. Y. R. (2000). The bHLH transcription factor hand2 plays parallel roles in zebrafish heart and pectoral fin development. Development 127, $2573-82$.

Zhang, X. Y. and Rodaway, A. R. F. (2007). SCL-GFP transgenic zebrafish: In vivo imaging of blood and endothelial development and identification of the initial site of definitive hematopoiesis. Dev. Biol. 307, 179-194.

Zhu, H., Traver, D., Davidson, A. J., Dibiase, A., Thisse, C., Thisse, B., Nimer, S. and Zon, L. I. (2005). Regulation of the lmo2 promoter during hematopoietic and vascular development in zebrafish. Dev Biol 281, 256-269.

\section{Author Contributions}

C.H, K.D.P., S.N., S.B, C.M. designed, performed, and interpreted zebrafish experiments; S.N., E.C., A.B. performed chicken experiments; K. P. performed lightsheet imaging with technical and equipment support by G.S., J.H; E.C.B, K.P., A.F. performed section-based zebrafish lineage tracing; K.W.R, P.M. provided and generated mutants and maternal-zygotic mutant zebrafish; H.J.P, M.B., R.K. designed, performed, and interpreted lamprey experiments; C.R., L.C. designed, performed, and interpreted Ciona experiments; I.K, Z.K designed, performed, and interpreted amphioxus experiments; C.H., K.P., and C.M. assembled and wrote the manuscript with contributions from all co-authors.

\section{Acknowledgements}

We thank Sibylle Burger and Seraina Bötschi for technical and husbandry support; the lab of Dr. Stephan Neuhauss for zebrafish husbandry support; the labs of Dr. Esther Stöckli and Dr. Jerome Gros for chicken experimentation support; the ZBM at UZH for imaging support; Dr. Fiona Wardle for input and support on the ChIP-seq panel in Fig. 4E; the lab of Dr. Magdalini Polymenidou for vibratome access; Karolína Ditrychová for cloning the pKD001 construct; Dr. Ashley Bruce, Dr. Rebecca Burdine, and Dr. Michael Tsang for sharing transcription factor constructs; the Stowers Institute histology facility for assistance with lamprey embryo sectioning; and all members of the Mosimann lab for constructive input. This work has been supported by a Swiss National Science Foundation (SNSF) professorship [PP00P3_139093] and SNSF R'Equip grant 150838 (Lightsheet Fluorescence Microscopy), a Marie Curie Career Integration Grant from the European Commission [CIG PCIG14-GA-2013-631984], the Canton of Zürich, the 
UZH Foundation for Research in Science and the Humanities, the Swiss Heart Foundation, and the ZUNIV FAN/UZH Alumni to C.M; a UZH CanDoc to C.H.; EuFishBioMed and Company of Biologists travel fellowships to K.D.P.; the Stowers Institute (grant \#1001) to H.J.P. and R.K.; NIH/NHLBI R01 award HL108643, trans-Atlantic network of excellence award 15CVD01 from the Leducq Foundation to L.C.; a long-term fellowship ALTF 1608-2014 from EMBO to C.R. 
Figures
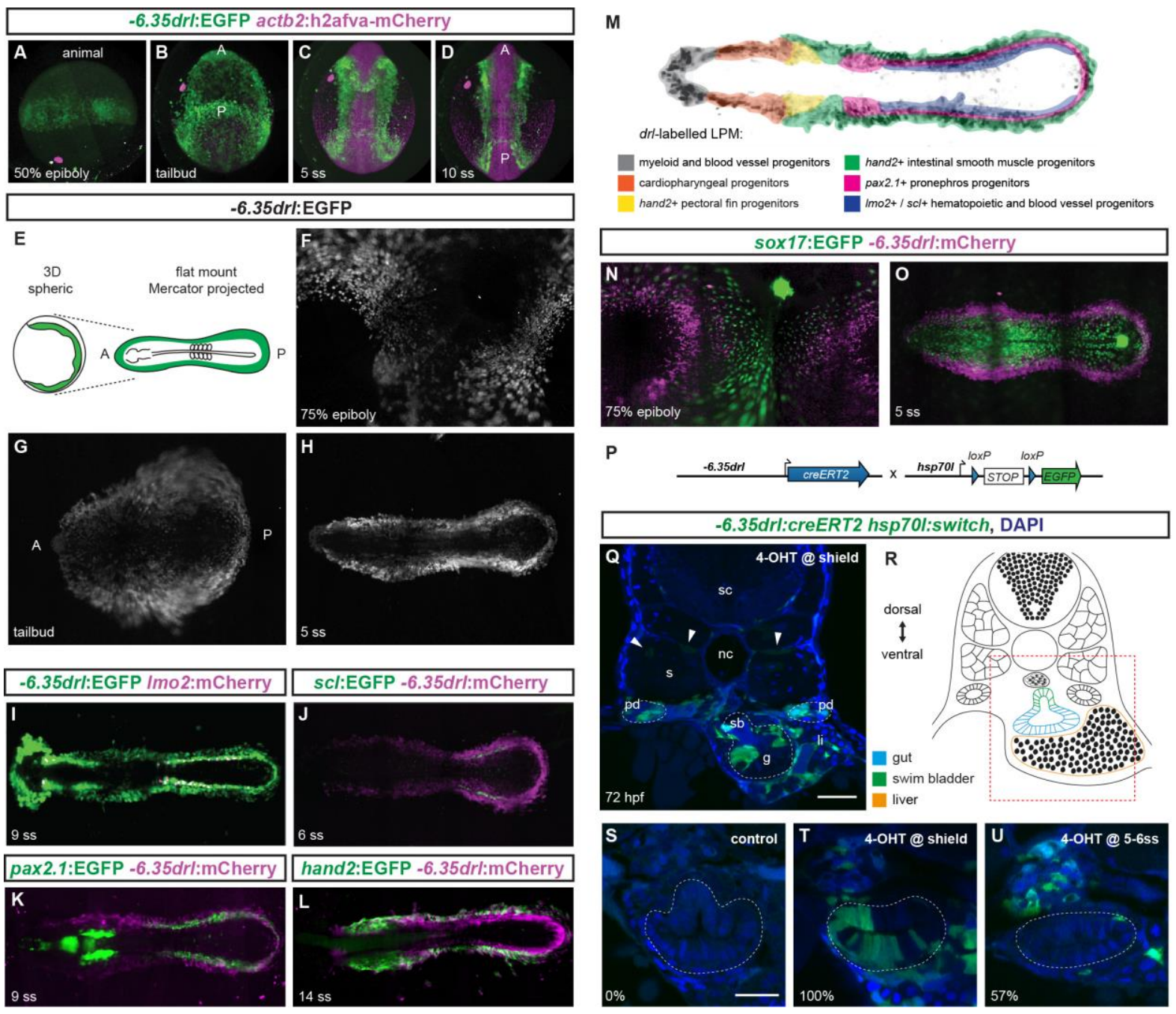

Figure 1: The LPM forms around the entire circumference of the developing zebrafish embryo. (A-D) Panoramic SPIM imaging of 50\% epiboly to 10 ss embryos transgenic for -6.35drl:EGFP (green) and actb2:h2afva-mCherry (nuclei marker, magenta); maximum-intensity-projected, lateral (A), dorsal (B-D) views. (E-O) Panoramic SPIM imaged zebrafish embryos shown as Mercator projections. (E) Schematic of Mercator projection to visualize entire embryo. (F-l) Single time point projections (dorsal views) of double-transgenics for $-6.35 \mathrm{drl}$ reporters and $l \mathrm{mo} 2, \mathrm{scl}$, pax2.1, or hand2 reporters co-expressed in dedicated LPM territories. (M) $6.35 \mathrm{drl}$ reporter embryo schematic, depicting LPM sub-territories at $10 \mathrm{ss}$. (N,O) Time lapse projections of $-6.35 \mathrm{drl}: \mathrm{mCherry}$ (magenta) with sox17:EGFP, showing LPM versus sox17-marked endoderm from gastrulation until 10 ss. (P) Schematic of 6.35drl:creERT2 $x$ hsp70l:switch cross for genetic lineage tracing. (Q) Representative whole-body transverse section of $3 \mathrm{dpf}$ $6.35 \mathrm{drl}$ lineage-traced embryo 4-OHT-induced at shield stage. Arrowheads depict rare trunk muscle labeling. (R) Schematic of transverse section with endoderm-derived organs at 3 dpf. (S-U) Transverse sections of -6.35drl lineage tracing, 4-OHT induction shield stage to 5-6 ss; note gradual loss of endoderm labeling (intestinal cells, dashed region). Numbers indicate percentage of embryos with intestinal lineage-labeling. Notochord (nc), somite (s), spinal cord (sc), pronephric duct (pd), liver (li), swim bladder (sb), and gut (g) labeled in (q), nuclei in blue (DAPI; Q,S-U). (F-O) Anterior to the left. Scale bar (Q) $50 \mu \mathrm{m}$ and (S-U) $25 \mu \mathrm{m}$. 


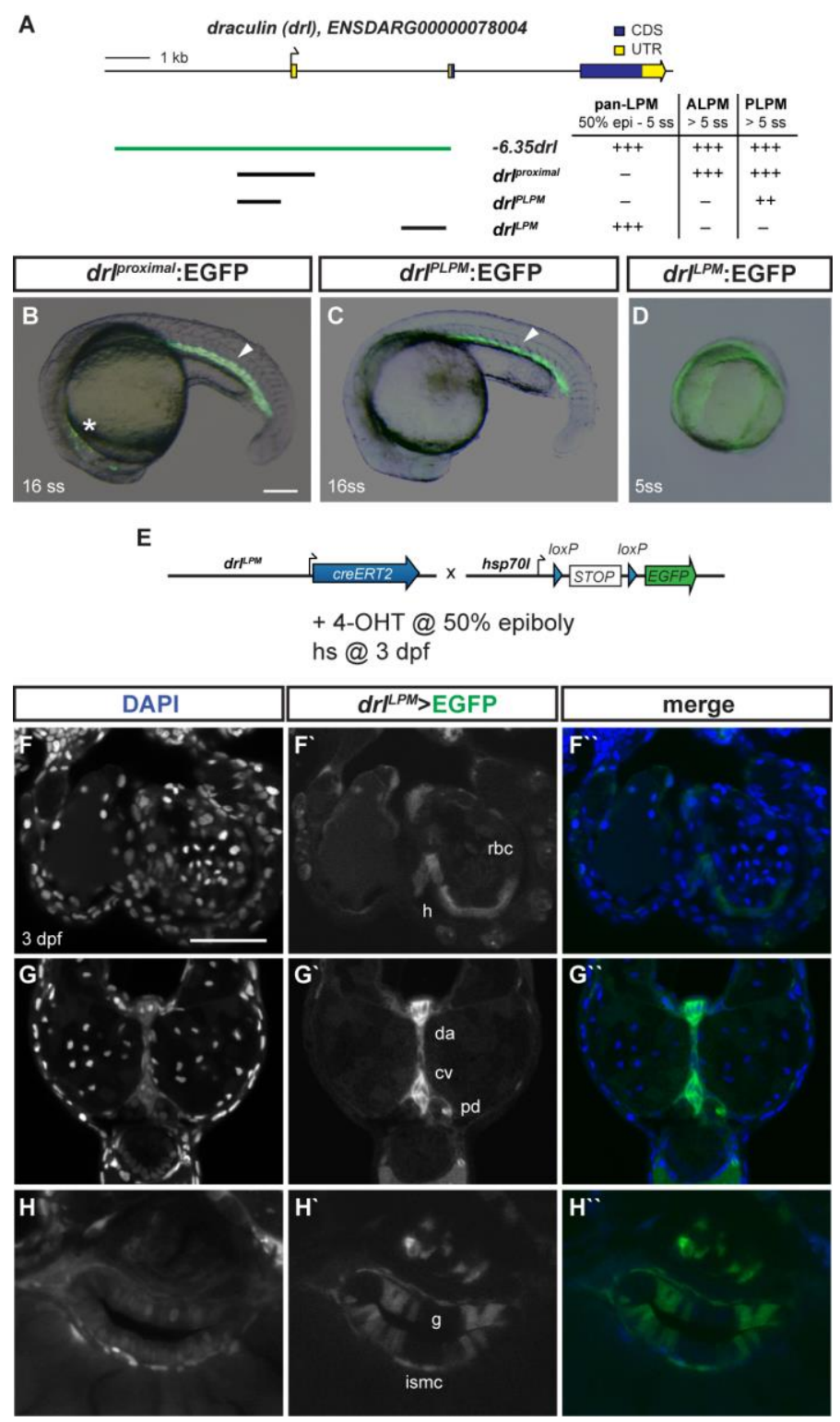

Figure 2: The -6.35drl cis-regulatory region contains an early pan-LPM enhancer. (A) Schematic of $d r l$ locus, $-6.35 d r l$ cisregulatory region (green), smaller isolated fragments $d r l^{\text {proximal }}, d r l^{P L P M}$, and $d r l^{L P M}$ with specific reporter activity. Table indicates pan-LPM expression (50\% to $5 \mathrm{ss}$ ) or ALPM/PLPM from 5 ss onwards, absent expression (-) to strong expression (+++). (B-D) Spatio-temporal expression of $d r l^{P L P M}, d r l^{\text {proximal }}$, and $d r l^{L P M}$ reporter transgenics; at $16 \mathrm{ss}, d r l^{P L P M}$ and $d r l^{\text {proximal }}$ express in PLPM (arrowheads in B,C), $d r l^{P L P M}$ additionally in ALPM (asterisk in B); note pan-LPM activity of $d r l^{L P M}(\mathbf{D})$. (E) Schematic representation of $d r l^{L P M}$ :creERT2 $x$ hsp70l:switch cross for genetic lineage tracing. (F-H) Transverse sections at $3 \mathrm{dpf}$ of $d r l^{L P M}$ :creERT2 lineage tracing after 4-OHT induction at shield stage results in specific labeling of LPM-derived tissue including heart (h), red blood cells $(\mathrm{rbc})$, dorsal aorta (da) and cardinal vein (cv) endothelium, pronephric duct (pd), and intestinal smooth muscle cells (ismc). $d r l^{L P M}$ :creERT2 also traces endoderm-derived tissue, shown for gut (g). Nuclei counterstained with DAPI (blue). Scale bars (B) 250 $\mu \mathrm{m}$ and (F) $50 \mu \mathrm{m}$. 

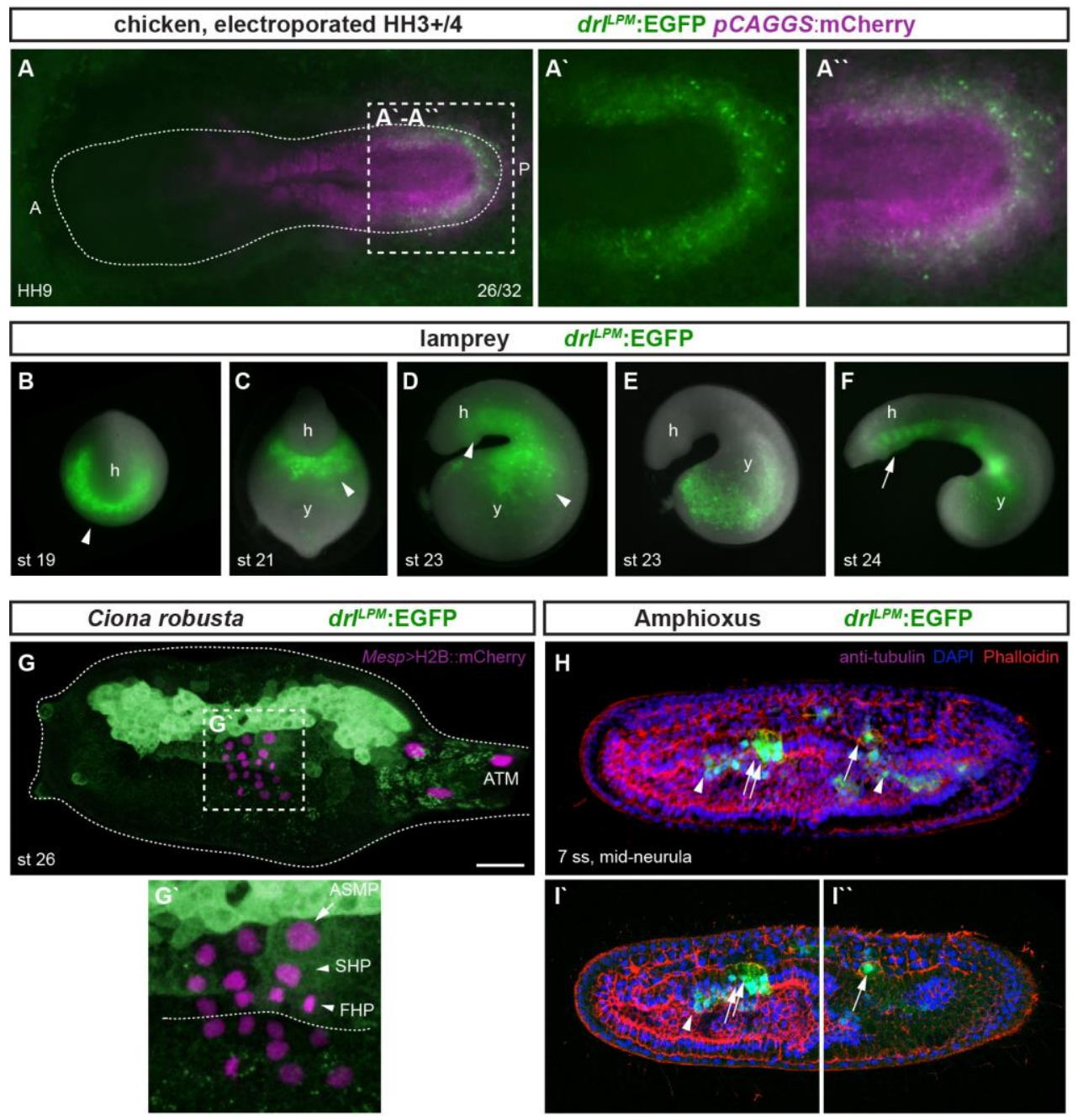

Figure 3: The zebrafish $d_{r l}^{L P M}$ cis-regulatory element reads out a LPM program in various chordates. (A) HH9-staged ex ovocultured chicken embryos electroporated at $\mathrm{HH} 3+/ 4$ with $d r l^{L P M}: E G F P$ and ubiquitous $p C A G G S: m C h e r r y$ control (magenta), showing specific $d r l^{L P M}$ reporter expression in the electroporated LPM. The dashed line depicts outline of chicken embryo, anterior to the left; zoom-in depicts boxed region in (A), single and merged channel (A', $\left.\mathbf{A}^{\prime \prime}\right)$. (B-F) Transient transgenic lamprey embryos with $d r l^{L P M}$ :EGFP expression in the anterior mesendoderm (arrowheads) and overlying the yolk at neurula stages (st 19-21) (B-C), and in the developing pharynx (arrow) during head protrusion (st 23-24) (C-F); views anterior (B), ventral (C), lateral (D-F), head (h) and yolk (y). (G) Ciona robusta embryos at 18 hpf (st 26) expressing $d r l^{L P M}$ :EGFP, with Mesp $>\mathrm{H} 2 \mathrm{~B}:$ :mCherry to track the B7.5 cardiopharyngeal cell lineage (magenta). EGFP is specifically expressed in mesenchymal lineages and partly overlaps with B7.5 derivatives including atrial siphon muscles precursors (ASMP) (white arrows) and both first and second heart precursors (FHPs and SHPs) (white arrowheads). Representative larva shown, zoom-in of boxed region in ( $\left.\mathbf{G}^{`}\right)$; dashed line indicates midline, anterior to the left, scale bar (G) $25 \mu \mathrm{m}$. (H) Confocal Z-stack of mid-neurula stage (7 ss) amphioxus embryo injected with $d r l^{L P M}: E G F P$, showing specific reporter activity in lateral endoderm (arrowhead), ventral half of somites (arrows), and evaginated somites (double arrows). Embryo counterstained with Phalloidin (red), DAPI (blue); lateral view as 3D-rendering (H) and Z-stack sagittal sections $\left(\mathbf{H}-\mathbf{H}^{\prime}\right)$, anterior to the left, dorsal to the top. 

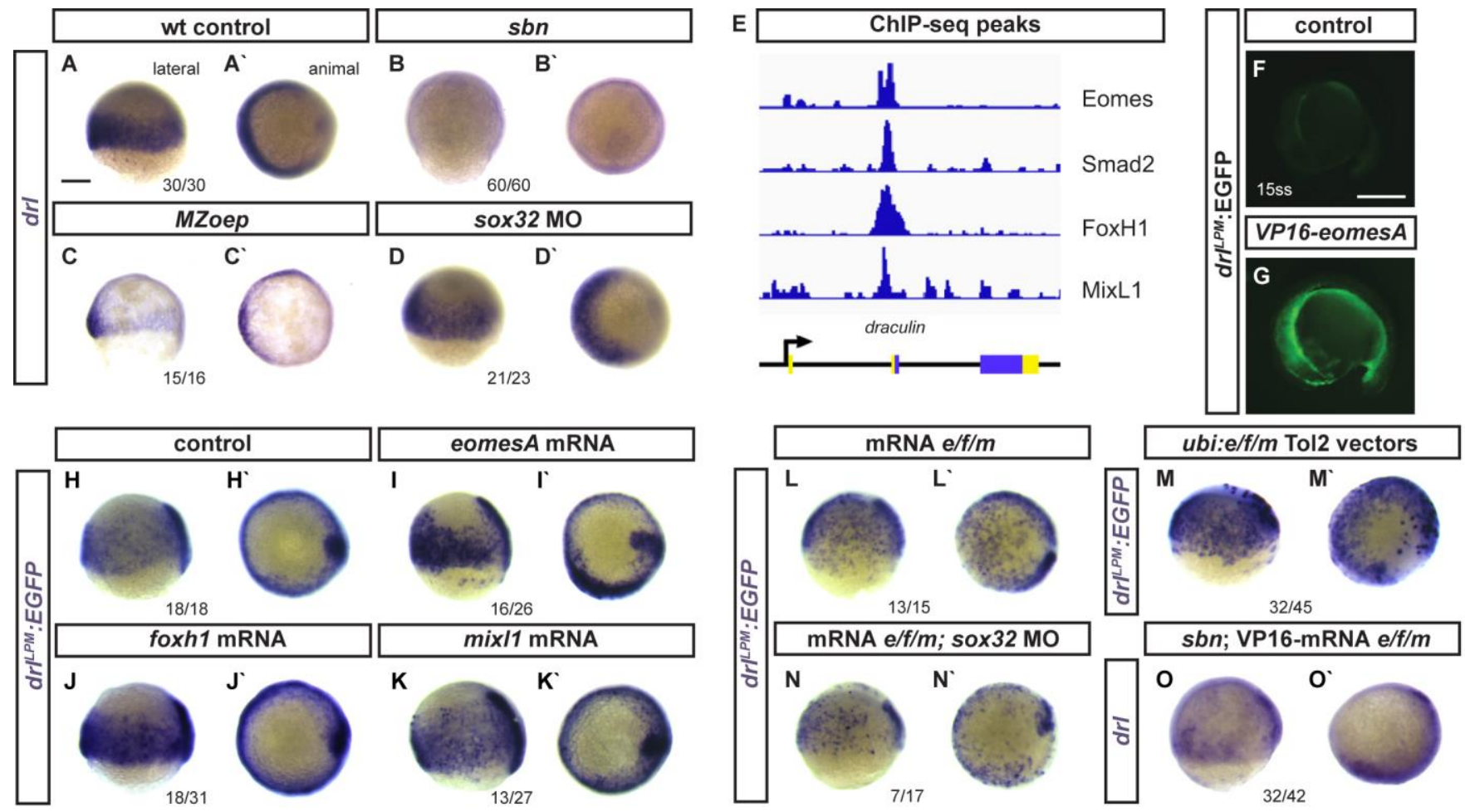

Figure 4: Eomes, FoxH1, and MixL1 induce $d r l^{L P M}$ activity. (A-D) mRNA ISH for endogenous $d r l$ expression in wildtype (wt), BMP-perturbed (sbn), Nodal-perturbed (MZoep), and endoderm-perturbed (sox32 morphant) backgrounds, shield stage; drl expression is strongly suppressed in $s b n$, dorsally lost in MZoep, and unaffected upon sox32 perturbation. (E) ChIP-seq peaks of Eomes, Smad2 (Nelson et al., 2014), FoxH1 (Charney et al., 2017), and MixL1 (Nelson et al., 2017) in the endogenous $d r l$ locus. $(\mathbf{F}, \mathbf{G})$ Microinjection of VP16-eomesA in $d r l^{L P M}: E G F P$ reporter transgenics enhances and prolongs EGFP expression in native $d r l$ expression domain. (H-K) EGFP ISH expression on $d r l^{L P M}$ :EGFP embryos injected with full-length eomesA, foxhl, or mixl1 shows enhanced reporter activity in the native LPM territory. (L-O) EGFP ISH in $d r l^{L P M}: E G F P$ embryos injected with eomesA (e), foxh1 $(f)$, and mixll $(\mathrm{m})(\mathrm{e} / \mathrm{f} / \mathrm{m})$ as mRNA $(\mathbf{L})$ or as $u b i$-driven DNA vectors $(\mathbf{M})$ show ectopic reporter expression, also when endoderm is perturbed in sox32 morphants (N). (O) ISH for endogenous $d r l$ in ventral BMP mutants $(s b n)$ injected with $e / f / m$ as constitutively active VP16 fusion ORFs, note a partial restoration of $d r l$ expression at dorsal Nodal-active margin. (A-D,H-O) Lateral and animal views shown at shield stage as indicated. Scale bar (A) $250 \mu \mathrm{m}$ and (F) $400 \mu \mathrm{m}$. 


\section{Material and Methods}

\section{Animal experiments and husbandry}

Zebrafish and chick experiments were carried out in accordance with the recommendations of the national authorities of Switzerland (Animal Protection Ordinance). The protocols and the experiments were approved by the cantonal veterinary office of the Canton Zurich (Kantonales Veterinäramt). Zebrafish care and all experimental procedures were carried out in accordance with the European Communities Council Directive (86/609/EEC), according to which all embryo experiments performed before 120 hours post fertilization are not considered animal experimentation and do not require ethics approval. Adult zebrafish for breeding were kept and handled according to animal care regulation of the Kantonales Veterinäramt Zürich (TV4209). All zebrafish (Danio rerio) were raised, kept, and handled as described (Westerfield, 2007). To assure same-staged embryos, adults were separated before mating by dividers. Embryos were collected and mixed to ensure a homologous diversity of clutches, and subsequently raised in E3 medium in temperature-controlled incubators at 21,28 , and $32^{\circ} \mathrm{C}$ respectively. Lamprey studies were conducted in accordance with the Guide for the Care and Use of Laboratory Animals of the National Institutes of Health, and protocols were approved by the Institutional Animal Care and Use Committees of the California Institute of Technology (Protocol \# 1436-11).

\section{Transgenic constructs and transgenic zebrafish lines}

The upstream cis-regulatory elements of the zebrafish $d r l$ gene (ENSDARG00000078004; ZDB-GENE991213-3) were amplified from zebrafish wildtype genomic DNA (Extended Data Table 1; regulatory elements) and TOPO-cloned into the $p E N T R^{\mathrm{TM}} 5^{\prime}-T O P O{ }^{\circledR} T A$ Cloning® plasmid (Invitrogen) according to the manufacturer's instructions.

Subsequent cloning reactions were performed with the Multisite Gateway system with LR Clonase II Plus (Life Technologies) according to the manufacturer's instructions. $\operatorname{drl}^{P L P M}: E G F P$ (pDestTol2pA2 $\left.d r l^{P L P M}: E G F P\right)$ and $d r l^{\text {proximal }}:$ EGFP $\left(p D e s t T o l 2 p A 2_{-} d r l^{\text {proximal }}: E G F P\right)$ were assembled from $\mathrm{pENTR} / 5^{\prime}, d r l^{P L P M}$ or $\mathrm{pENTR} / 5^{\prime}, d r l^{\text {proximal }}$ together with Tol2kit vectors \#383 (pME-EGFP), \#302 (p3E_SV4ŌpolyA), and \#394 (pDestTol2A2) as backbone. drl ${ }^{L P M}: E G F P\left(p D e s t T o l 2 p A 2 \_d r l^{L P M}: E G F P\right)$ and $d r l^{\min L P M}: E G F P\left(p D e s t T o l 2 p A 2_{-} d r l^{\min L P M}: E G F P\right)$ were assembled from $\mathrm{pENTR} / 5^{\prime}{ }_{-} d r l^{L P M}$ or $\mathrm{pENTR} / 5$ ' $d r l^{\text {minLPM }}$ together with $p M E$ - $\beta$-globin_minpromoter_EGFP (Tamplin et al., 2015), Tol2kit vectors \#302 (p3E_SV40polyA), and \#394 (pDestTol2A2) as backbone (Kwan et al., 2007). drl ${ }^{\text {LPM }}$ :creERT2 $\left(p D e s t T o l 2 C Y \_d r l^{L P M}:\right.$ creERT2,alpha-crystallin:YFP) and -5.0sox17:creERT2 (pDestTol2CY_5.0sox 17:creERT2,alpha-crystallin:YFP) were assembled from $p E N T R / 5^{\prime}{ }^{\prime} d r l^{L P M}$ or $p E N T R / 5^{\prime}{ }_{-}-5.0$ sox 17 together with $p C M 293$ ( $\left.p E N T R / D \_c r e E R T 2\right)(M o s i m a n n$ et al., 2011), Tol2kit vector \#302 (p3E_SV40polyA), and $p C M 326$ (pDestTol2CY) as backbone (Mosimann et al., 2015).

The regulatory elements of mouse-specific LPM enhancers were PCR-amplified (Extended Data Table 1; regulatory elements), TOPO-cloned into the $p E N T R 5^{\prime}$ plasmid, and assembled together with $p K D 001$ ( $p M E$ $\beta$-globin_minpromoter_EGFP with improved Kozak sequence), Tol2kit vectors \#302 (p3E_SV40polyA), and \#394 (pDestTol2A2) as backbone.

Assembled reporter constructs were injected at a concentration of $25 \mathrm{ng} / \mu \mathrm{L}$ together with $25 \mathrm{ng} / \mu \mathrm{L}$ Tol2 mRNA for Tol2-mediated zebrafish transgenesis (Felker and Mosimann, 2016). Injected F0 founders were screened for specific EGFP or alpha-crystallin:YFP expression. Fish were raised to adulthood and screened in F1 for germline transmission. Single-insertion transgenic strains were generated, and microscopy images were taken on a Leica M205FA with a Leica DFC450C digital camera or a Leica SP8 confocal microscope with Plan-Apochromat 20x/0.5 objective. Images were processed using Leica LAS and Fiji.

Established transgenic and mutant lines used in this study included -6.35drl:EGFP (Mosimann et al., 2015), -6.35drl:mCherry ${ }^{z h 705}$ (Sánchez-Iranzo et al., 2018), -6.35drl:creERT2 (Mosimann et al., 2015), ubi:creERT2 (Mosimann et al., 2011), ubi:switch (Mosimann et al., 2011), hsp70l:switch ${ }^{z h 701}$, lmo2:dsRED2 (Zhu et al., 2005), scl/tall:EGFP (Jin et al., 2006), pax2.1:EGFP (Picker et al., 2002), hand2:EGFP (Yin et al., 2010), 
actb2:h2afva-mCherry (Krens et al., 2011), maternal and zygotic EGF-CFC co-receptor oep gene mutants (MZoep) (Gritsman et al., 1999), and somitabun ${ }^{d t c 24}$ mutants (sbn, smad5) (Hild et al., 1999; Mullins et al., 1996).

\section{Zebrafish CreERT2-based lineage tracing}

Lineage tracing experiments were performed by crossing female hsp70l:switch or ubi:switch (Mosimann et al., 2011) reporter carriers with the male creERT2 drivers -6.35drl:creERT2 (Mosimann et al., 2015), sox 17:creERT2, and $d r l^{L P M}$ :creERT2. Embryos were induced using 4-Hydroxytamoxifen (4-OHT) (Cat\#H7904; Sigma H7904) from fresh or pre-heated $\left(65^{\circ} \mathrm{C}\right.$ for $\left.10 \mathrm{~min}\right)$ stock solutions in DMSO at a final concentration of $10 \mu \mathrm{M}$ in E3 embryo medium at indicated time points (Felker et al., 2016). Embryos were washed in untreated E3 medium at $24 \mathrm{hpf}$. To induce EGFP transcription in hsp70l:switch carrying embryos, the embryos were incubated at $37^{\circ} \mathrm{C}$ for 1 hour, 3 hours before fixation. Embryos were fixed with $4 \%$ Paraformaldehyde (PFA) overnight at $4^{\circ} \mathrm{C}$ at $3 \mathrm{dpf}$ and processed for confocal analysis.

\section{Zebrafish transverse vibratome sections}

Transverse sections were generated as previously described (Gays et al., 2017). Fixed embryos were washed in PBS, embedded in 6\% low-melting agarose (Sigma-Aldrich) in PBS/0.1\% Tween-20 (Sigma-Aldrich), and cut into $130 \mu \mathrm{m}$ thick sections using a vibratome (Leica VT 1000S). Sections were mounted in DAPIcontaining Vectashield (Cat\#H-1200; Vector Laboratories). Sections were analyzed with a Zeiss LSM710 confocal microscope with a Plan-Apochromat 40x/1.3 oil DIC M27 objective. Images were cropped and adjusted for brightness using ImageJ/Fiji (Schindelin et al., 2012). Graphs were generated in Graphpad Prism 5 .

\section{Zebrafish gene knockdown experiments}

The previously established sox32 morpholino (Sakaguchi et al., 2001) was synthesized by GeneTools (sequence: 5'-TGCTTTGTCATGCTGATGTAGTGGG-3') and dissolved in nuclease-free water to a stock concentration of $10 \mu \mathrm{g} / \mu \mathrm{l}$. The sox32 morpholino injection mix of $4 \mu \mathrm{g} / \mu \mathrm{l}$ was incubated for $10 \mathrm{~min}$ at $65^{\circ} \mathrm{C}$, and $8 \mathrm{ng}$ were microinjected into one-cell stage zebrafish embryos.

\section{Zebrafish CRISPR-Cas9 site-directed mutagenesis}

sgRNAs were obtained in an oligo-based approach as previously described (Burger et al., 2016). Briefly, primer extension was performed using Phusion polymerase (NEB) of forward primer 5'GAAATTAATACGACTCACTATAGG-N20-GTTTTAGAGCTAGAAATAGC-3' (including $20 \mathrm{nt}$ target site) and invariant reverse primer

5 AAAAGCACCGACTCGGTGCCACTTTTTCAAGTTGATAACGGACTAGCCTTATTTTAACTTGCTATTTCTA GCTCTAAAAC-3' (PAGE-purified) (Bassett et al., 2013). The following target sites are shown in this manuscript: 5'-AGATTTGTTTAGTCAGTGTC-3' (drl ccG), 5'-GTCTGGAAACAGTCTGAATC-3' (drl ccH), 5'-GAGACTTCGCCCTTCGGTTC-3' (mixll ccB), and 5'-GACAGAACAGGCCACGTTGA-3' (mezzo ccA).

In vitro transcription of sgRNAs was performed as previously described (Burger et al., 2016) using MAXIscript T7 (Ambion). Afterwards, RNA was precipitated with ammonium acetate, washed with $75 \%$ ethanol, and dissolved in DEPC water. RNA quality was checked on a MOPS gel. RNPs of Cas9 protein (Cas9mCherry, pMJ293 (available from Addgene)) (Burger et al., 2016) and sgRNA were in vitro-assembled for 10 min at $37^{\circ} \mathrm{C}$ in $300 \mathrm{mM} \mathrm{KCl}$ to ensure maximum cleavage efficiency and microinjected into the cell of onecell stage embryos (Burger et al., 2016). The CRISPR target regions in the transgenic locus were amplified using specific target region amplification primers (Table S1; target region amplifications).

Sequencing analysis was performed with the R software package CrispRVariants. CrispRVariants enables variant counting and visualization (Lindsay et al., 2015). 


\section{Zebrafish overexpression experiments}

For early developmental transcription factor genes, ORFs were PCR-amplified from mixed-stage zebrafish cDNA using ORF-specific primers (Table S1; coding sequence primers); full-length zebrafish eomesA was derived from I.M.A.G.E clone IRBOp991A0739D (Source BioScience LifeSciences, UK). All CDS were TOPO-cloned into the $p E N T R / D^{\mathrm{TM}}$ Directional TOPO ${ }^{\circledR}$ plasmid (Invitrogen) according to the manufacturer's instructions. Subsequent cloning reactions were performed with the Multisite Gateway system with LR Clonase II Plus (Invitrogen) according to the manufacturer's instructions and as previously described (Felker and Mosimann, 2016). The $C D S$ as pENTR/D vectors were assembled with $p E N T R 5^{\prime}$ _ubi (Mosimann et al., 2011), pENTR5'_T7-VP16, pENTR5'_T7-eng or pENTR5'_T7 together with Tol2kit vectors \#302 (p3E_SV40polyA), and \#394 (pDestTol2A2) as backbone (Kwan et al., 2007). Afterwards, plasmids were linearized (in case of T7-VP16, T7-eng or T7) and in vitro transcribed (Roche). The EomesA-VP16 plasmid (containing 153aa-431aa of the zebrafish EomesA ORF) (Bruce et al., 2003) was kindly provided by Dr. Rebecca Burdine. The plasmid was linearized via NotI followed by SP6 in vitro transcription. The Ntl-VP16 plasmid was kindly provided by Dr. Ashley Bruce. The etv5a-VP16 and etv5a-eng plasmids were kindly provided by Dr. Michael Tsang. All mRNAs were precipitated with ammonium acetate, washed with $75 \%$ ethanol, and dissolved in DEPC water. mRNA quality was checked on MOPS gels as described (Burger et al., 2016).

\section{Zebrafish whole-mount in situ hybridization}

Total RNA was extracted from zebrafish embryos from various stages during development. This RNA was used as template for generation of first-strand complementary DNA (cDNA) by Superscript III First-Strand Synthesis kit (Cat\#18080051; Invitrogen). In situ hybridization (ISH) probes were designed with an oligonucleotide-based method (including T7 promoter added to the reverse primers) using zebrafish cDNA (Table S1; in situ hybridization probes). The following oligonucleotide pairs (including T7 promoter added to the reverse primers) were used to amplify the DNA template from zebrafish cDNA. The ISH probe for $a d m p$ was obtained from pCS2_ADMP plasmid, and sizzled from pCS2_Sizzled. Admp and sizzled were linearized by ClaI. The gata $2 a$ probe was obtained from the middle entry vector $p C M 238$. For in vitro transcription, T7 RNA polymerase (Roche) and digoxygenin (DIG)-labeled NTPs (Roche) were used. Afterwards, RNA was precipitated with lithium chloride, washed with $75 \%$ ethanol, and dissolved in DEPC water. RNA quality was checked on a MOPS gel. ISH on whole-mount zebrafish embryos was executed as described before (Thisse and Thisse, 2008). After ISH, embryos were transferred to 80-95\% glycerol (Sigma-Aldrich), and microscopy images were taken on a Leica M205FA with a Leica DFC450C digital camera. Images were cropped and adjusted for brightness using ImageJ/Fiji.

\section{Zebrafish selective plan illumination microscopy (SPIM)}

At $30-50 \%$ epiboly, embryos in the chorion were embedded into $1 \%$ low-melting agarose with optional 0.016\% Ethyl 3-aminobenzoate methanesulfonate salt (Tricaine, Cat\#A5040; Sigma) in E3 embryo medium, and sucked into a FEP tube (inner diameter: $2.0 \mathrm{~mm}$, wall thickness: $0.5 \mathrm{~mm}$ ). 6-7 embryos were positioned on top of each other. The FEP tube was mounted in the microscope imaging chamber filled with E3 medium. Panoramic (3D) SPIM/lightsheet microscopy and subsequent image processing (Mercator projections) were performed as previously described (Schmid et al., 2013). A z-stack of 402 planes was obtained from every embryo with an interval of 2 min for a period of 14-17 hours. Images were processed using Leica LAS, ImageJ/Fiji, and Photoshop CS6.

\section{Chicken embryo incubation and ex evo culturing}

Ex ovo culturing was adapted from previously established protocols (New, 1955). Fertilized chicken eggs were obtained from a local hatchery and stored at $12^{\circ} \mathrm{C}$ up to maximum of 14 days. Prior to use, eggs were incubated horizontally for 17 hours until Hamburger-Hamilton $(\mathrm{HH}) 3+/ 4$ in a $39^{\circ} \mathrm{C}$ incubator with $55-65 \%$ humidity. After incubation, the eggs were kept for at least $30 \mathrm{~min}$ at RT before opening. Eggs were opened in 
a petri dish and a layer of thick albumin together with the chalaziferous layer was removed using a plastic Pasteur pipette. A paper ring was placed around the embryo on the yolk and dissection scissors were used to cut the yolk membrane around the ring. The paper ring with the embryo was cleaned from remaining yolk and transferred and placed upside down on a semisolid albumin/agarose $(43.5 \mathrm{ml}$ thin albumin incubated for 2 hours at $55^{\circ} \mathrm{C}, 5 \mathrm{ml} 2 \%$ agarose, $1.5 \mathrm{ml} 10 \%$ glucose in $30 \mathrm{~mm}$ petri dishes) culturing plate. Embryos were recovered for at least 2 hours at RT before electroporation.

\section{Chicken embryo injection and electroporation}

For electroporations, a customized electroporation chamber was used containing an electrode with a positive pole on the bottom of the chamber and separate negative electrode on a holder (kindly provided by the lab of Dr. Jerome Gros). Both electrodes were mounted and connected to a square wave electroporator (BTX ECM 830). The electroporation chamber was filled with HBSS (Gibco Life Technologies), and the embryocontaining paper ring was placed in the chamber with the dorsal side up. The DNA mixture was injected by a mouth injector along the primitive streak beneath the pellucid membrane. The positive electrode holder was placed on top of the streak to allow electricity pulses flow through the embryo ( 3 pulses, $8 \mathrm{~V} \times 50$ milliseconds, 500 milliseconds interval). The embryos were placed back on the albumin culturing plates with the ventral side up and placed back at $39^{\circ} \mathrm{C}$ until HH8-9. Microscopy images of the embryos were taken at HH8-9 on a Leica M205FA with a Leica DFC450C digital camera. Images were cropped and adjusted for brightness using ImageJ/Fiji.

All injection mixtures for electroporations contained $0.1 \%$ fast green dye, $0.1 \%$ methyl-cellulose, $300 \mathrm{ng} / \mu \mathrm{l}$ control plasmid $p C A G G s$ ( $p C M V: H 2 B-C A G G-R F P$, abbreviated for chicken $\beta$-actin promoter CAGG$m$ Cherry) and $1 \mu \mathrm{g} / \mu \mathrm{l}$ of the plasmid of interest.

\section{Lamprey experiments}

The $d r l^{L P M}$ and $d r l^{\min L P M}$ regulatory elements were amplified from the zebrafish vectors $d r l^{L P M}: E G F P$ and $d r l^{\min L P M}: E G F P$ by PCR using KOD Hot Start Master Mix (Novagen) (Table S1; regulatory elements). The amplified enhancers were cloned into the HLC vector for lamprey transgenesis (Parker et al., 2014b), containing the mouse $c$-Fos minimal promoter, by Gibson assembly using the Gibson Assembly Master Mix (NEB).

Injections for I-SceI meganuclease-mediated lamprey transient transgenesis were performed using $P$. marinus embryos at the one-cell stage with injection mixtures containing $0.5 \mathrm{U} / \mu \mathrm{l}$ I-SceI enzyme and $20 \mathrm{ng} / \mu \mathrm{l} \mathrm{reporter}$ construct as described previously (Parker et al., 2014a). Selected EGFP-expressing embryos were fixed in MEM-FA and dehydrated in methanol for in situ hybridization. EGFP-expressing embryos were imaged using a Zeiss SteREO Discovery V12 microscope with variable zoom and a Zeiss Axiocam MRm camera with AxioVision Rel 4.6 software. Images were cropped and adjusted for brightness using Adobe Photoshop CS5.1.

For mRNA ISH, total RNA was extracted from st 21-26 P. marinus embryos using the RNAqueous Total RNA Isolation Kit (Ambion). This was used as a template for 3' rapid amplification of cDNA ends (RACE) with the GeneRacer Kit and SuperScript III RT (Invitrogen). A 339bp-long pmHandA in situ probe was designed based on a characterized cDNA sequence from the closely related Arctic lamprey (Lethenteron camtschaticum) (Kuraku et al., 2010), and this sequence was amplified by PCR from 3' RACE cDNA using KOD Hot Start Master Mix (Novagen) with the following primers: 5'-GCGGAGGACATTGAGCATC-3' (forward) and 5'-TGGAATTCGAGTGCCCACA-3' (reverse). The cDNA fragment was cloned into the $p C R 4$ TOPO vector (Invitrogen). The 709bp-long eGFP probe was described previously (Parker et al., 2014b).

DIG-labelled probes were generated and used in lamprey whole-mount ISH as described previously(SaukaSpengler et al., 2007). Embryos were cleared in 75\% glycerol and imaged using a Leica MZ APO microscope with variable zoom and Lumenera Infinity 3 camera with Lumenera Infinity Capture v6.5.3 software. Images were cropped and adjusted for brightness using Adobe Photoshop CS5.1. 
After ISH, selected embryos were transferred into 30\% sucrose in PBS, embedded in O.C.T. Compound (Tissue-Tek), and cut into $10 \mu \mathrm{m}$-thick cryosections using a CryoStar NX70 cryostat (Thermo Scientific). Images were taken using a Zeiss Axiovert 200 microscope with AxioCam HRc camera and AxioVision Rel 4.8.2 software.

\section{Ciona experiments}

The regulatory elements $-6.35 \mathrm{drl}$ and $d r l^{L P M}$ were amplified from the zebrafish vectors $d r l^{L P M}: E G F P$ and $d r l^{\operatorname{minLPM}}: E G F P$ and subcloned in a vector upstream of $u n c 76: G F P$ (Table S1; regulatory elements).

Gravid Ciona robusta adults were obtained from M-REP (San Diego CA, USA). To test the activity of the zebrafish enhancers in Ciona robusta, $80 \mu \mathrm{g}$ of $d r l^{L P M}: E G F P$ or $d r l^{\min L P M}: E G F P$ were injected in a mixture with the reporter plasmid Mesp (Davidson et al., 2005) to mark the B7.5 cardiopharyngeal lineage with H2B:mCherry $(10 \mu \mathrm{g})$.

For antibody staining, embryos were fixed in 4\% MEM-PFA for 30 min, rinsed several times in PBT (PBS/0.1\% Tween-20), and incubated with anti-GFP (1:500, Mouse mAb, Roche) with 2\% normal goat serum in PBT at $4{ }^{\circ} \mathrm{C}$ overnight. Embryos were washed in PBT and then incubated with donkey anti-mouse secondary antibody (1:1000) coupled to Alexa Fluor 488 (Life Technologies) in PBT with 2\% normal goat serum for 2 hours at RT, then washed in PBT(Racioppi et al., 2014).

\section{Amphioxus experiments}

The regulatory elements $-6.35 d r l$ and $d r l^{L P M}$ were amplified from the zebrafish vectors $-6.35 d r l: E G F P$ and $d r l^{L P M}: E G F P$ and subcloned upstream of a EGFP reporter in the pPB vector carrying PiggyBac transposon terminal repeats (Kozmikova and Kozmik, 2015). Adults of Branchiostoma lanceolatum were collected in Banyuls-sur-mer, France, prior to the summer breeding season and raised in the laboratory until spawning. The spawning of amphioxus male and females was induced by shifting of the temperature as described (Fuentes et al., 2007). For microinjection of amphioxus eggs, a mixture of $p P B-6.35 d r l: E G F P$ or $p P B-d r l^{L P M}: E G F P(200$ $\mathrm{ng} / \mu \mathrm{l})$ with PiggyBac transposase mRNA $(100 \mathrm{ng} / \mu \mathrm{l})$ in $15 \%$ glycerol was used. Transgenic embryos were allowed to develop until neurula stage, fixed in $4 \%$ PFA overnight at $4{ }^{\circ} \mathrm{C}$, mounted with Vectashield with DAPI (Vector Laboratories), and analyzed by Leica SP5 confocal microscope. The confocal images were adjusted for brightness and contrast with ImageJ/Fiji.

\section{Table S1: Primer sequences}

\begin{tabular}{|c|c|}
\hline regulatory elements & \\
\hline$d r l^{P L P M}$ zebrafish & $\begin{array}{l}\text { 5'-TTTCAATTTCAAAGGAGC-3' } \\
\text { 5'-TGTTTTGAGTGAGTCGGTTAGG-3' }\end{array}$ \\
\hline$d r l^{\text {proximal }}$ zebrafish & $\begin{array}{l}\text { 5'-TTTCAATTTCAAAGGAGC-3' } \\
\text { 5'-CAACTGGGACATATTTAAACC-3' }\end{array}$ \\
\hline $\boldsymbol{d r l}^{L P M}$ zebrafish & $\begin{array}{l}\text { 5'-CACTTCCTAATCTGCTATTGCC-3' } \\
\text { 5'-CCAAGTGTGAATTGGGATCG-3' }\end{array}$ \\
\hline for lamprey expression & $\begin{array}{l}\text { 5'-CCCTCGAGGTCGACGCACTTCCTAATCTGCTATTG-3', } \\
\text { 5'- GAGGATATCGAGCTCGCCAAGTGTGAATTGGGATC-3, }\end{array}$ \\
\hline for ciona expression & $\begin{array}{l}\text { 5'-CTCGAGCACTTCCTAATCTGCTATTGCC-3' (w/Xhol) } \\
\text { 5'-TCTAGACCAAGTGTGAATTGGGATCG-3' (w/Xbal) }\end{array}$ \\
\hline$d r l^{\min L P M}$ & 5'-GTCAAATCAATCAATGCGGTGC-3' \\
\hline
\end{tabular}


bioRxiv preprint first posted online Feb. 7 2018: doi: $h$ ttp $/ / d x$ doi.org/10.1101/261115. The copyright holder for this preprint (which was not peer-reviewed) is the author/funder, who has granted bioRxiv a license to display the preprint in perpetuity. It is made available under a CC-BY-NC-ND 4.0 International license.

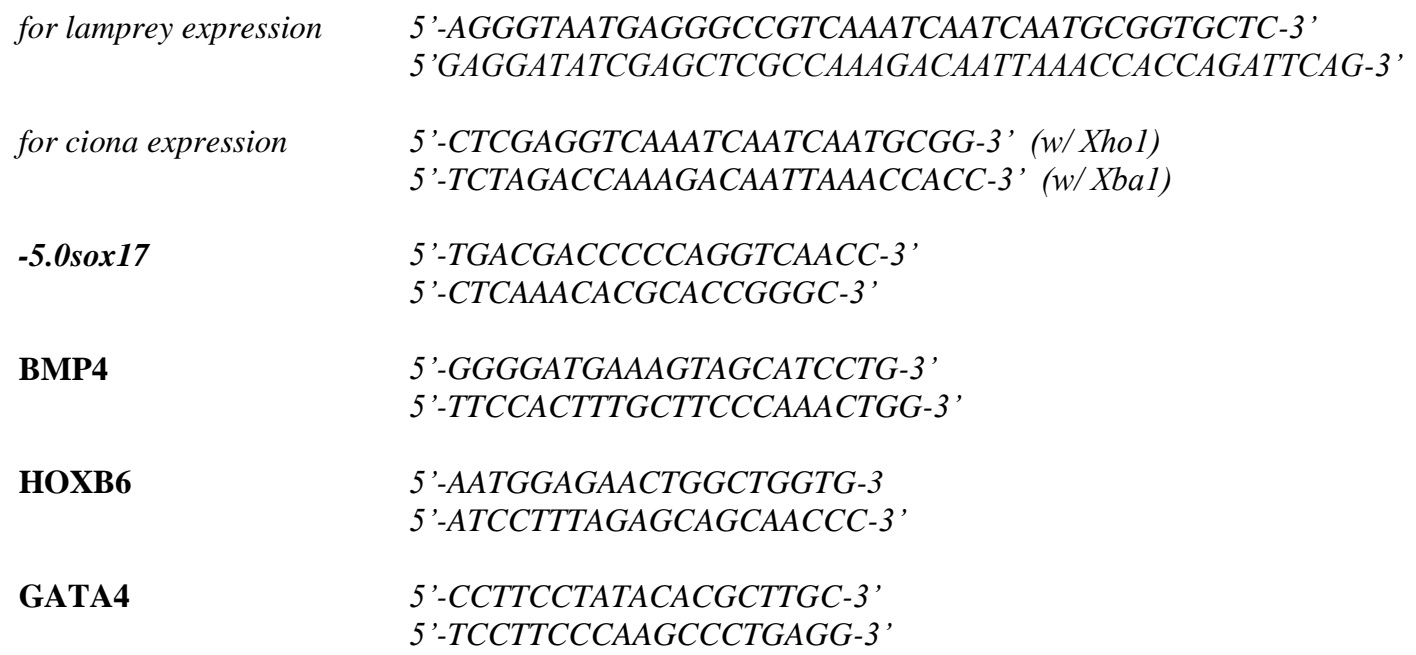

\begin{tabular}{|c|c|}
\hline \multicolumn{2}{|c|}{ target region amplification } \\
\hline$d r l$ & $\begin{array}{l}\text { 5'-AATCACAAAGTCTGGGTTAACAGAG-3' } \\
\text { 5'-CGACCAGGATGGGCACCAC-3'(EGFP region) }\end{array}$ \\
\hline mixl1 & 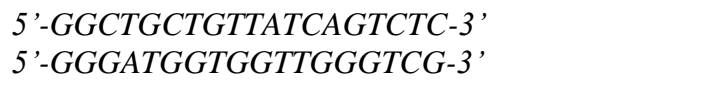 \\
\hline mezzo & 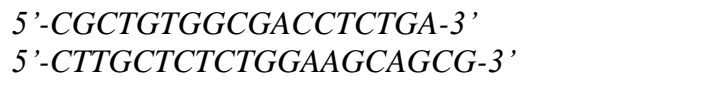 \\
\hline
\end{tabular}

\begin{tabular}{|c|c|}
\hline coding sequence & \\
\hline foxh1 & $\begin{array}{l}\text { 5'-CACCATGACAAAGCACTGGGGG-3' } \\
\text { 5'-TTAAAGAGAATATTTGCAAAGG-3' }\end{array}$ \\
\hline mixl1 & $\begin{array}{l}\text { 5'-CACCATGGCAGTCGTGCACGGAAACC-3' } \\
\text { 5'-CTAAATGAAGCCATTAAAACC-3' }\end{array}$ \\
\hline spr2 & $\begin{array}{l}\text { 5'-CACCATGGCTGCATTAACCTTATCC-3, } \\
\text { 5'-TTACTCTCTCTTGATGTTGCTC-3, }\end{array}$ \\
\hline osrl & $\begin{array}{l}\text { 5'-CACCATGGGTAGTAAGACGCTCC -3' } \\
\text { 5'-TTACTTTATCTTGGCTGGC-3' }\end{array}$ \\
\hline mespaa & $\begin{array}{l}\text { 5'-CACCATGGACGCCTCCACG-3' } \\
\text { 5'-TCAGCACTGTCCATGGAA-3' }\end{array}$ \\
\hline scl/tall & $\begin{array}{l}\text { 5`-CACCATGATGGAAAAACTGAAATCC-3`} \\
5^{`}-T C A C C G C T G G G C A T T T C-3 ’\end{array}$ \\
\hline
\end{tabular}


bioRxiv preprint first posted online Feb. 7, 2018; doi: http://dx doi.org/10.1101/261115. The copyright holder for this preprint (which was not peer-reviewed) is the author/funder, who has granted bioRxiv a license to display the preprint in perpetuity. It is made available under a CC-BY-NC-ND 4.0 International license.

\begin{tabular}{|c|c|}
\hline$E G F P$ & $\begin{array}{l}\text { 5'-ATGGTGAGCAAGGGCGAGGAGC-3' } \\
\text { 5'-TAATACGACTCACTATAGGG-3' }\end{array}$ \\
\hline$d r l$ & $\begin{array}{l}\text { 5'-ATGAAGAATACAACAAAACCC-3' } \\
\text { 5'-TAATACGACTCACTATAGGTGAGAAGCTCTGGCCGC-3' }\end{array}$ \\
\hline hand2 & $\begin{array}{l}\text { 5'-CACCATGAGTTTAGTTGGAGGGTTTCC-3' } \\
\text { 5'-TAATACGACTCACTATAGGGTCATTGCTTCAGTTCCAATGCC-3', }\end{array}$ \\
\hline foxf1 & $\begin{array}{l}\text { 5'-ATACCGCGCTATCATACC-3' } \\
\text { 5'-GAATTCTAATACGACTCACTATAGGGAAAGCAGTCTTAACTCTGG-3' }\end{array}$ \\
\hline ved & $\begin{array}{l}\text { 5'-CACCATGAAGGGTCAGTTCTCC-3' } \\
\text { 5'-TAATACGACTCACTATAGGGCACAATAAAGCCAGTCC-3', }\end{array}$ \\
\hline tmem88a & $\begin{array}{l}\text { 5'-ATGAGTCTTCCACGAAACGG-3' } \\
\text { 5'-TAATACGACTCACTATAGGGATGAAGCACAGGCCC-3, }\end{array}$ \\
\hline
\end{tabular}




\section{References}

Bassett, A. R., Tibbit, C., Ponting, C. P. and Liu, J.-L. (2013). Highly efficient targeted mutagenesis of Drosophila with the CRISPR/Cas9 system. Cell Rep. 4, 220-8.

Bruce, A. E. E., Howley, C., Zhou, Y., Vickers, S. L., Silver, L. M., King, M. Lou and Ho, R. K. (2003). The maternally expressed zebrafish T-box gene eomesodermin regulates organizer formation.

Development 130, 5503-5517.

Burger, A., Lindsay, H., Felker, A., Hess, C., Anders, C., Chiavacci, E., Zaugg, J., Weber, L. M. L. M., Catena, R., Jinek, M., et al. (2016). Maximizing mutagenesis with solubilized CRISPR-Cas9 ribonucleoprotein complexes. Development 143, 2025-37.

Davidson, B., Shi, W. and Levine, M. (2005). Uncoupling heart cell specification and migration in the simple chordate Ciona intestinalis. Development 132,

Felker, A. and Mosimann, C. (2016). Contemporary zebrafish transgenesis with Tol2 and application for Cre/lox recombination experiments. Methods Cell Biol. 135, 219-44.

Felker, A., Nieuwenhuize, S., Dolbois, A., Blazkova, K., Hess, C., Low, L. W. L. W. L., Burger, S., Samson, N., Carney, T. J. T. J., Bartunek, P., et al. (2016). In Vivo Performance and Properties of Tamoxifen Metabolites for CreERT2 Control. PLoS One 11, e0152989.

Fuentes, M., Benito, E., Bertrand, S., Paris, M., Mignardot, A., Godoy, L., Jimenez-Delgado, S., Oliveri, D., Candiani, S., Hirsinger, E., et al. (2007). Insights into spawning behavior and development of the european amphioxus (Branchiostoma lanceolatum). J. Exp. Zool. Part B Mol. Dev. Evol. 308B, 484-493.

Gays, D., Hess, C., Camporeale, A., Ala, U., Provero, P., Mosimann, C. and Santoro, M. M. (2017). An exclusive cellular and molecular network governs intestinal smooth muscle cell differentiation in vertebrates. Development 144, dev.133926.

Gritsman, K., Zhang, J., Cheng, S., Heckscher, E., Talbot, W. S. and Schier, A. F. (1999). The EGFCFC Protein One-Eyed Pinhead Is Essential for Nodal Signaling. 97, 121-132.

Hild, M., Dick, A., Rauch, G. J., Meier, A., Bouwmeester, T., Haffter, P. and Hammerschmidt, M. (1999). The smad5 mutation somitabun blocks Bmp2b signaling during early dorsoventral patterning of the zebrafish embryo. Development 126, 2149-59.

Jin, H., Xu, J., Qian, F., Du, L., Tan, C. Y., Lin, Z., Peng, J. and Wen, Z. (2006). The 5' zebrafish scl promoter targets transcription to the brain, spinal cord, and hematopoietic and endothelial progenitors. Dev Dyn 235, 60-67.

Kozmikova, I. and Kozmik, Z. (2015). Gene regulation in amphioxus: An insight from transgenic studies in amphioxus and vertebrates. Mar. Genomics 24, 159-166.

Krens, S. F. G., Möllmert, S. and Heisenberg, C.-P. (2011). Enveloping cell-layer differentiation at the surface of zebrafish germ-layer tissue explants. Proc. Natl. Acad. Sci. U. S. A. 108, E9-10; author reply E11.

Kuraku, S., Takio, Y., Sugahara, F., Takechi, M. and Kuratani, S. (2010). Evolution of oropharyngeal patterning mechanisms involving Dlx and endothelins in vertebrates. Dev. Biol. 341, 315-23.

Kwan, K. M., Fujimoto, E., Grabher, C., Mangum, B. D., Hardy, M. E., Campbell, D. S., Parant, J. M., Yost, H. J., Kanki, J. P. and Chien, C. Bin (2007). The Tol2kit: a multisite gateway-based construction kit for Tol2 transposon transgenesis constructs. Dev Dyn 236, 3088-3099.

Lindsay, H., Burger, A., Felker, A., Hess, C., Zaugg, J., Chiavacci, E., Anders, C., Jinek, M., Mosimann, C. and Robinson, M. D. (2015). CrispRVariants: precisely charting the mutation spectrum in genome engineering experiments. Cold Spring Harbor Labs Journals.

Mosimann, C., Kaufman, C. K. C. K., Li, P., Pugach, E. K. E. K., Tamplin, O. J. O. J. and Zon, L. I. L. I. (2011). Ubiquitous transgene expression and Cre-based recombination driven by the ubiquitin promoter in zebrafish. Development 138, 169-177. 
Mosimann, C., Panáková, D., Werdich, A. A. A., Musso, G., Burger, A., Lawson, K. L. K. L., Carr, L. A. L. A., Nevis, K. R. K. R., Sabeh, M. K. K., Zhou, Y., et al. (2015). Chamber identity programs drive early functional partitioning of the heart. Nat. Commun. 6, 8146.

Mullins, M. C., Hammerschmidt, M., Kane, D. A., Odenthal, J., Brand, M., van Eeden, F. J., FurutaniSeiki, M., Granato, M., Haffter, P., Heisenberg, C. P., et al. (1996). Genes establishing dorsoventral pattern formation in the zebrafish embryo: the ventral specifying genes. Development 123, 81-93.

New, D. A. T. (1955). A New Technique for the Cultivation of the Chick Embryo in vitro. Development 3,.

Parker, H. J., Sauka-Spengler, T., Bronner, M. and Elgar, G. (2014a). A reporter assay in lamprey embryos reveals both functional conservation and elaboration of vertebrate enhancers. PLoS One $\mathbf{9}$, e85492.

Parker, H. J., Bronner, M. E. and Krumlauf, R. (2014b). A Hox regulatory network of hindbrain segmentation is conserved to the base of vertebrates. Nature advance on,.

Picker, A., Scholpp, S., Böhli, H., Takeda, H. and Brand, M. (2002). A novel positive transcriptional feedback loop in midbrain-hindbrain boundary development is revealed through analysis of the zebrafish pax2.1 promoter in transgenic lines. Development 129, 3227-39.

Racioppi, C., Kamal, A. K., Razy-Krajka, F., Gambardella, G., Zanetti, L., di Bernardo, D., Sanges, R., Christiaen, L. A. and Ristoratore, F. (2014). Fibroblast growth factor signalling controls nervous system patterning and pigment cell formation in Ciona intestinalis. Nat. Commun. 5, 4830.

Sakaguchi, T., Kuroiwa, A. and Takeda, H. (2001). A novel sox gene, 226D7, acts downstream of Nodal signaling to specify endoderm precursors in zebrafish. Mech. Dev. 107, 25-38.

Sánchez-Iranzo, H., Galardi-Castilla, M., Minguillón, C., Sanz-Morejón, A., González-Rosa, J. M., Felker, A., Ernst, A., Guzmán-Martínez, G., Mosimann, C. and Mercader, N. (2018). Tbx5a lineage tracing shows cardiomyocyte plasticity during zebrafish heart regeneration. Nat. Commun. 9 , 428.

Sauka-Spengler, T., Meulemans, D., Jones, M. and Bronner-Fraser, M. (2007). Ancient Evolutionary Origin of the Neural Crest Gene Regulatory Network. Dev. Cell 13, 405-420.

Schindelin, J., Arganda-Carreras, I., Frise, E., Kaynig, V., Longair, M., Pietzsch, T., Preibisch, S., Rueden, C., Saalfeld, S., Schmid, B., et al. (2012). Fiji: an open-source platform for biological-image analysis. Nat. Methods 9, 676-82.

Schmid, B., Shah, G., Scherf, N., Weber, M., Thierbach, K., Campos, C. P., Roeder, I., Aanstad, P. and Huisken, J. (2013). High-speed panoramic light-sheet microscopy reveals global endodermal cell dynamics. Nat Commun 4, 2207.

Tamplin, O. J., Durand, E. M., Carr, L. A., Childs, S. J., Hagedorn, E. J., Li, P., Yzaguirre, A. D., Speck, N. A. and Zon, L. I. (2015). Hematopoietic stem cell arrival triggers dynamic remodeling of the perivascular niche. Cell 160, 241-52.

Thisse, C. and Thisse, B. (2008). High-resolution in situ hybridization to whole-mount zebrafish embryos. Nat. Protoc. 3, 59-69.

Westerfield, M. (2007). The Zebrafish Book: a guide for the laboratory use of zebrafish (Danio rerio). 5th ed. Eugene: University of Oregon Press.

Yin, C., Kikuchi, K., Hochgreb, T., Poss, K. D. and Stainier, D. Y. R. (2010). Hand2 regulates extracellular matrix remodeling essential for gut-looping morphogenesis in zebrafish. Dev. Cell 18, 973-984.

Zhu, H., Traver, D., Davidson, A. J., Dibiase, A., Thisse, C., Thisse, B., Nimer, S. and Zon, L. I. (2005). Regulation of the lmo 2 promoter during hematopoietic and vascular development in zebrafish. Dev Biol 281, 256-269. 


\section{Supplementary Figures}
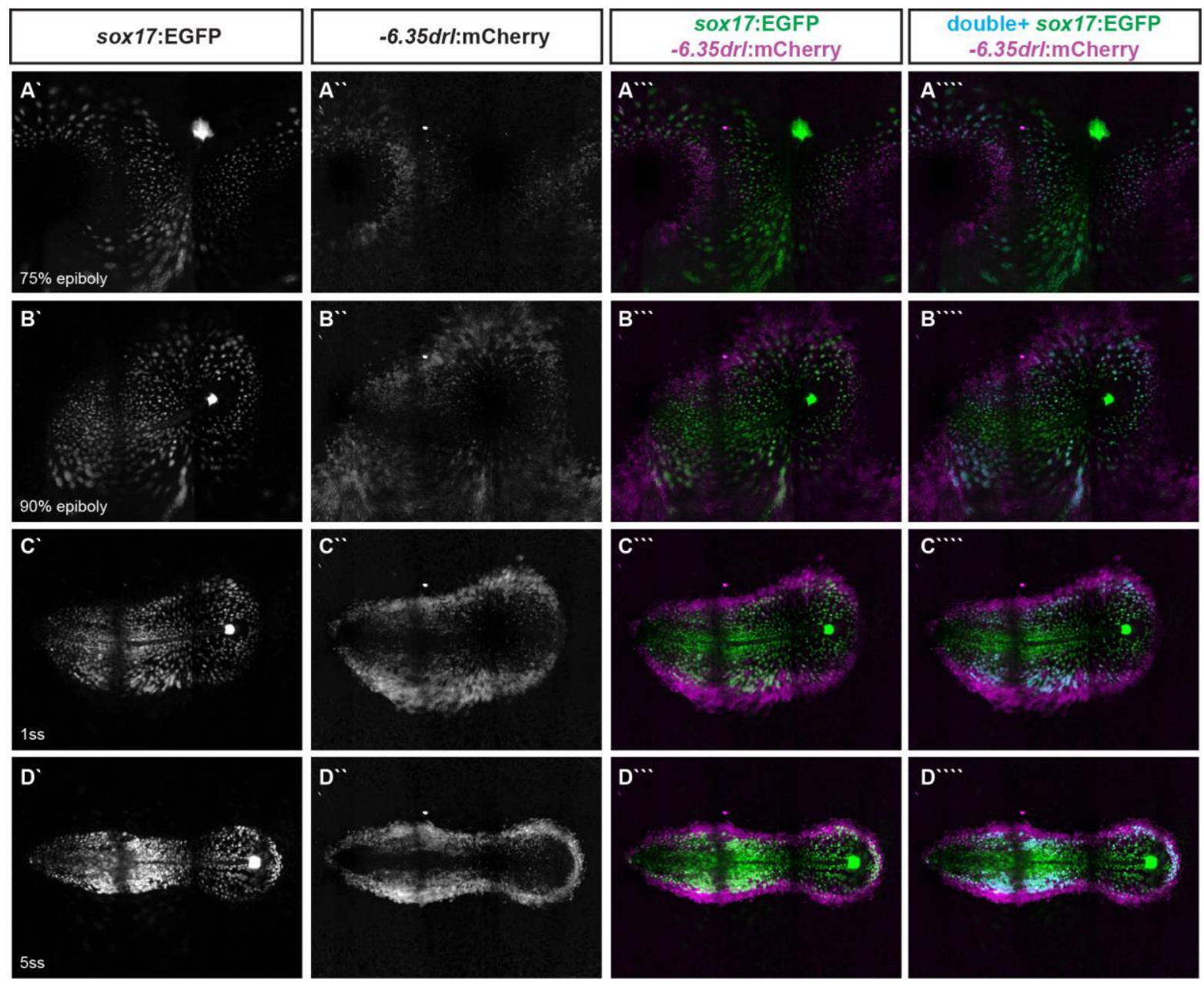

Fig. S1

-6.35drl reporter expression overlaps with sox17-marked endoderm during gastrulation. (A-D) Supporting Mercator projections of the SPIM time-lapse imaging of -6.35drl:mCherry (magenta) combined with sox 17:EGFP to visualize the $d r l$-expressing emerging cell population versus endoderm from $70 \%$ epiboly until 5 ss (see also Movies S3 and S4). (A`-D“,A“-D”) Single and (A“'-D“') merged channels are shown. Double-positive cells are labelled in blue in (A“'”-D“'”). Of note, the blue labeling in the 5 ss embryo in panel (D'.') represents not per se double-positive cells, but the overlap between the endodermal and $d r l$-positive cell layer. 

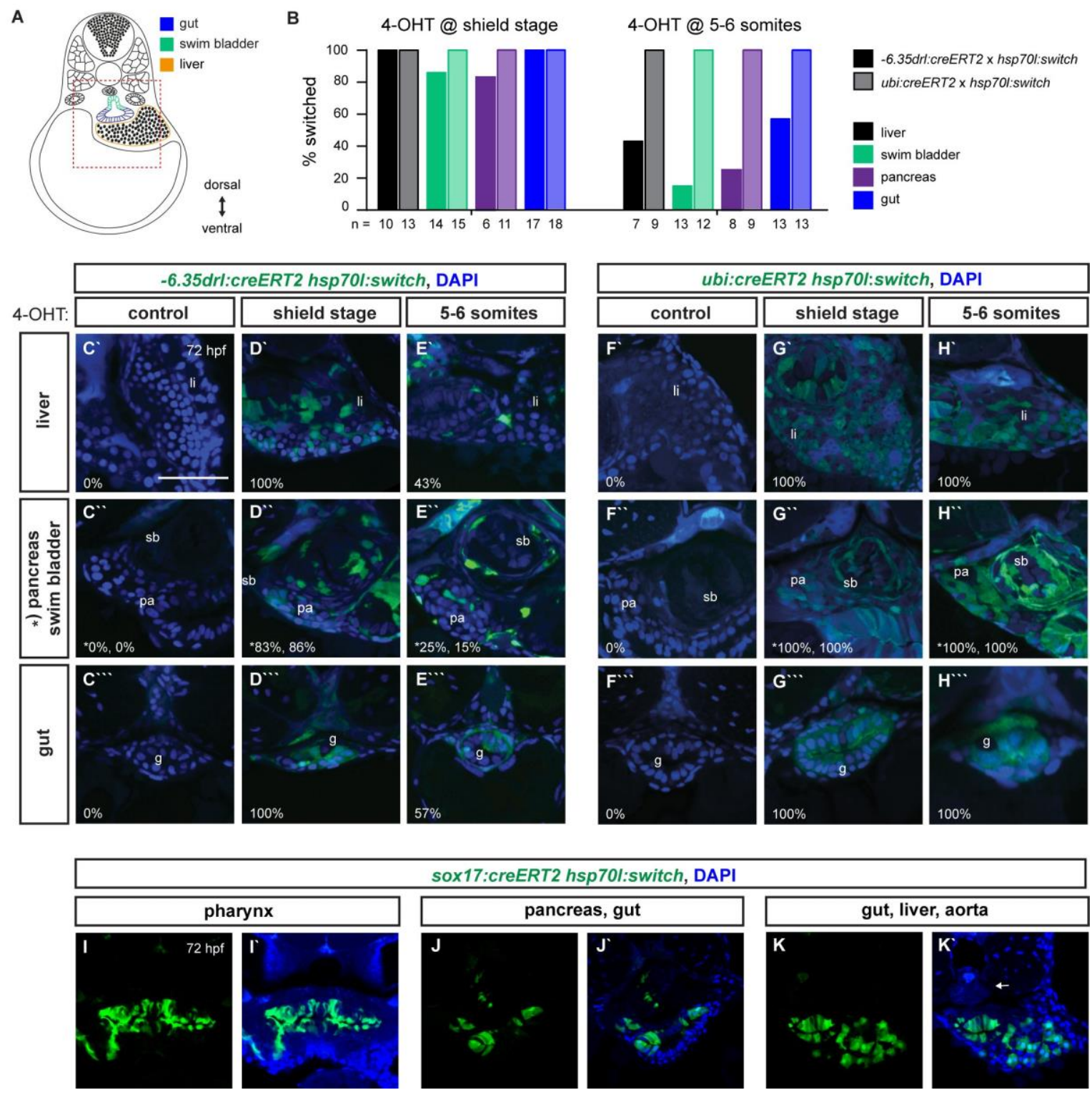

Fig. S2

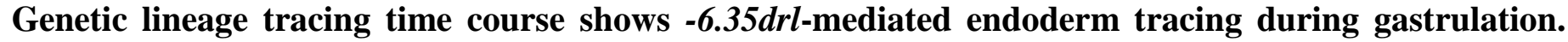
sox17-based lineage tracing exclusively labels endoderm-derived structures.

(A) Schematic representation of a transverse section indicating endoderm-derived organs. (B-H) Whole-body transverse sections of -6.35drl:creERT2- (C-E) or ubi:creERT2-lineage traced cells (F-H) at different 4-OHT induction time points (shield and 5-6 ss) to trigger hsp70l:switch recombination (green). (B) Quantification of observed lineage contribution at individual time points; 4-OHT induction at shield stage traces, besides of LPM-derived tissue, a high percentage of endoderm-derived tissue, as depicted for liver (li), swim bladder (sb), pancreas (pa), and gut (g), while 4-OHT induction at 5-6 ss traces shows a strong reduction in percentage of endoderm-derived tissue. No tissue bias in lineage reporter labeling could be detected for the hsp70l:switch line, as shown using the ubiquitous ubi:creERT2 (F-H). (I-K) Confocal transverse sections of sox 17:creERT2 lineage-traced endoderm cells after 4-OHT induction at shield stage and heat shock and fixation at $3 \mathrm{dpf}$. Lineage labeling is confined to endodermal lineages and absent from mesodermal and specifically LPM lineages, as depicted for the pharynx, pancreas, gut, and liver, in contrast to LPM-derived tissue such as the aorta (arrow in $\mathbf{K}^{\prime}$ ). Nuclei were counterstained with DAPI (blue). Numbers indicate the percentage of embryos showing lineage-labeling in depicted organs. Scale bar $50 \mu \mathrm{m}$. 


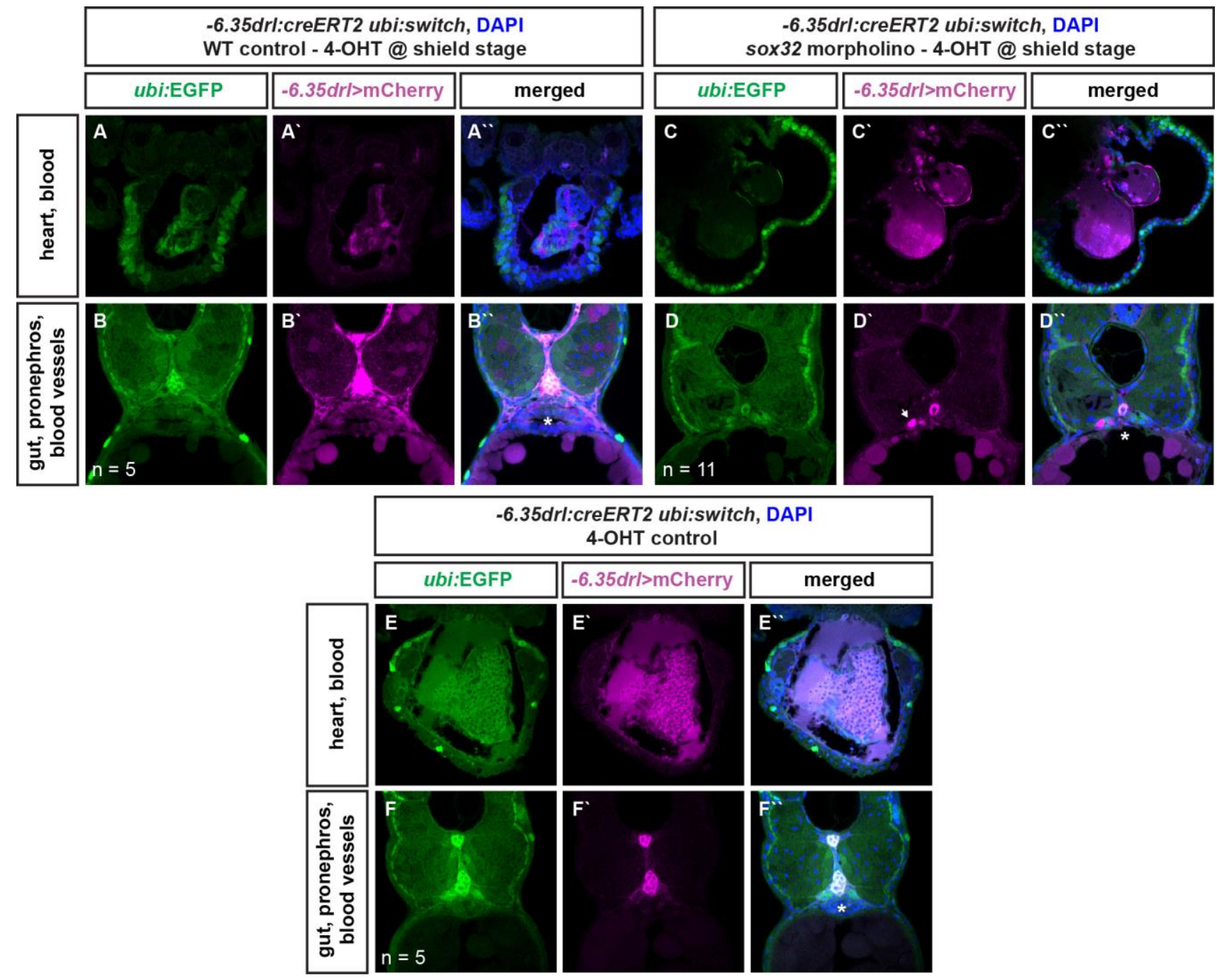

Fig. S3

LPM lineages and organs still arise in embryos devoid of endoderm. Confocal transverse sections of 6.35drl:creERT2 x ubi:switch embryos fixed at $3 \mathrm{dpf}$. (A-B) Embryos were induced with 4-OHT at shield stage, bringing $m$ Cherry under control of the $u b i$ promoter in cells with active CreERT2 at shield stage (magenta). LPM-derived organs, as depicted for heart, blood, pectoral fins, pronephros, and endothelial cells, and endoderm-derived lineages, including swim bladder, gut epithelium, and liver, are lineage-traced. (C-D) In sox32 morphants that are devoid of endoderm, 4-OHT induction at shield stage traced LPM-derived organs, as depicted for heart, blood, pectoral fins, pronephros (arrow), and endothelial cells. (E-F) Control without 4OHT admission reveals absence of any background activity of the used reporter (note autofluoresence of blood), confirming absence of leakiness of the creERT and loxP lines used. Asterisks indicate endodermderived gut epithelium $\left(\mathbf{B}^{\prime \prime}, \mathbf{F}^{\prime \prime}\right)$ or absence thereof (D“). Nuclei counterstained with DAPI (blue). 
bioRxiv preprint first posted online Feb. 7, 2018; doi: http://dx.doi.org/10.1101/261115. The copyright holder for this preprint (which was not peer-reviewed) is the author/funder, who has granted bioRxiv a license to display the preprint in perpetuity. It is made available under a CC-BY-NC-ND 4.0 International license.
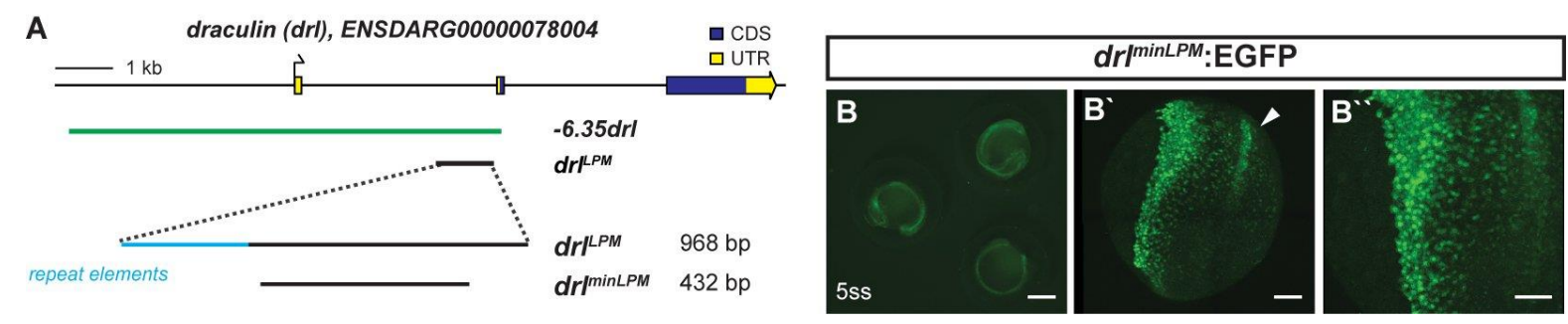

Fig. $\mathbf{S 4}$

Trimming of $\boldsymbol{d r l}^{L P M}$ to the minimal $432 \mathrm{bp}$ pan-LPM $\boldsymbol{d r} l^{\min L P M}$ enhancer region. (A) Schematic representation of the $-6.35 \mathrm{drl}$ locus including the PCR-based trimming approach to map the smallest LPM specific regulatory enhancer region; repetitive elements highlighted in blue. (B) Expression of $d r l^{\text {minLPM }}: E G F P$ at $5 \mathrm{ss}$; arrow indicates axial mesoderm expression. Scale bar $(\mathbf{B}) 400 \mu \mathrm{m}\left(\mathbf{B}^{\prime}\right)$ and $80 \mu \mathrm{m}\left(\mathbf{B}^{\prime \prime}\right)$. 
A

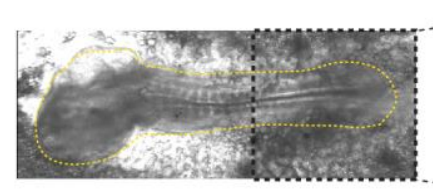

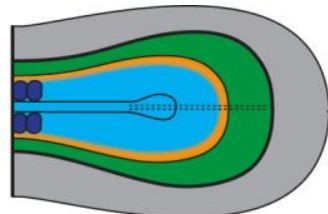

somites

pre-somitic mesoderm and notochord

intermediate mesoderm

lateral plate mesoderm

extra-embryonic mesoderm

primitive streak

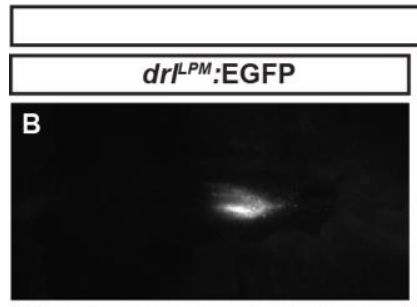

chicken HH9, electroporated $\mathrm{HH} 3+/ 4$
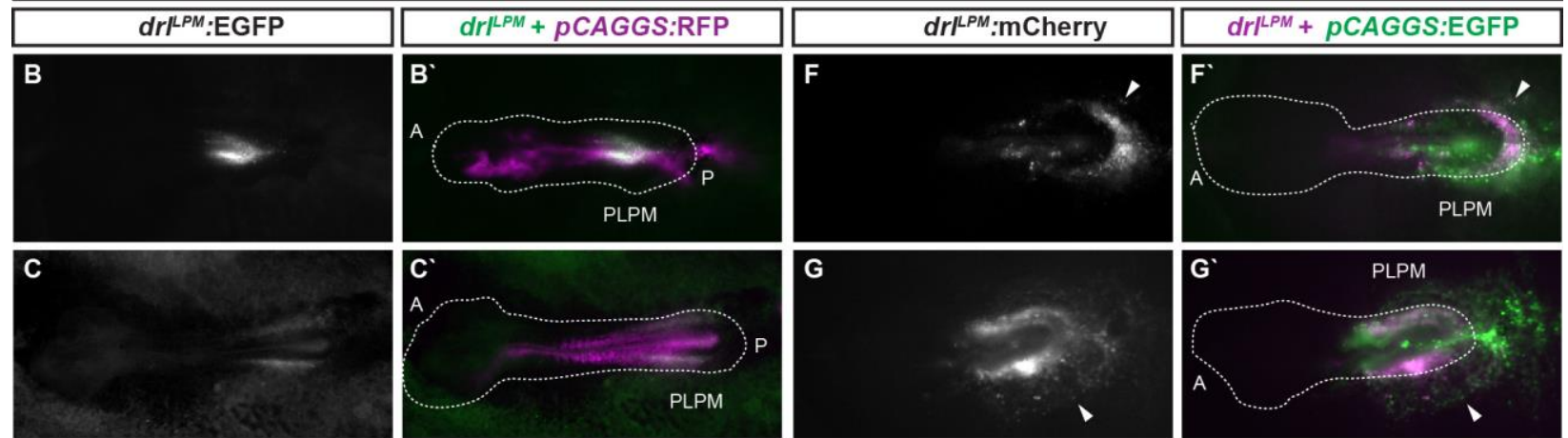

G
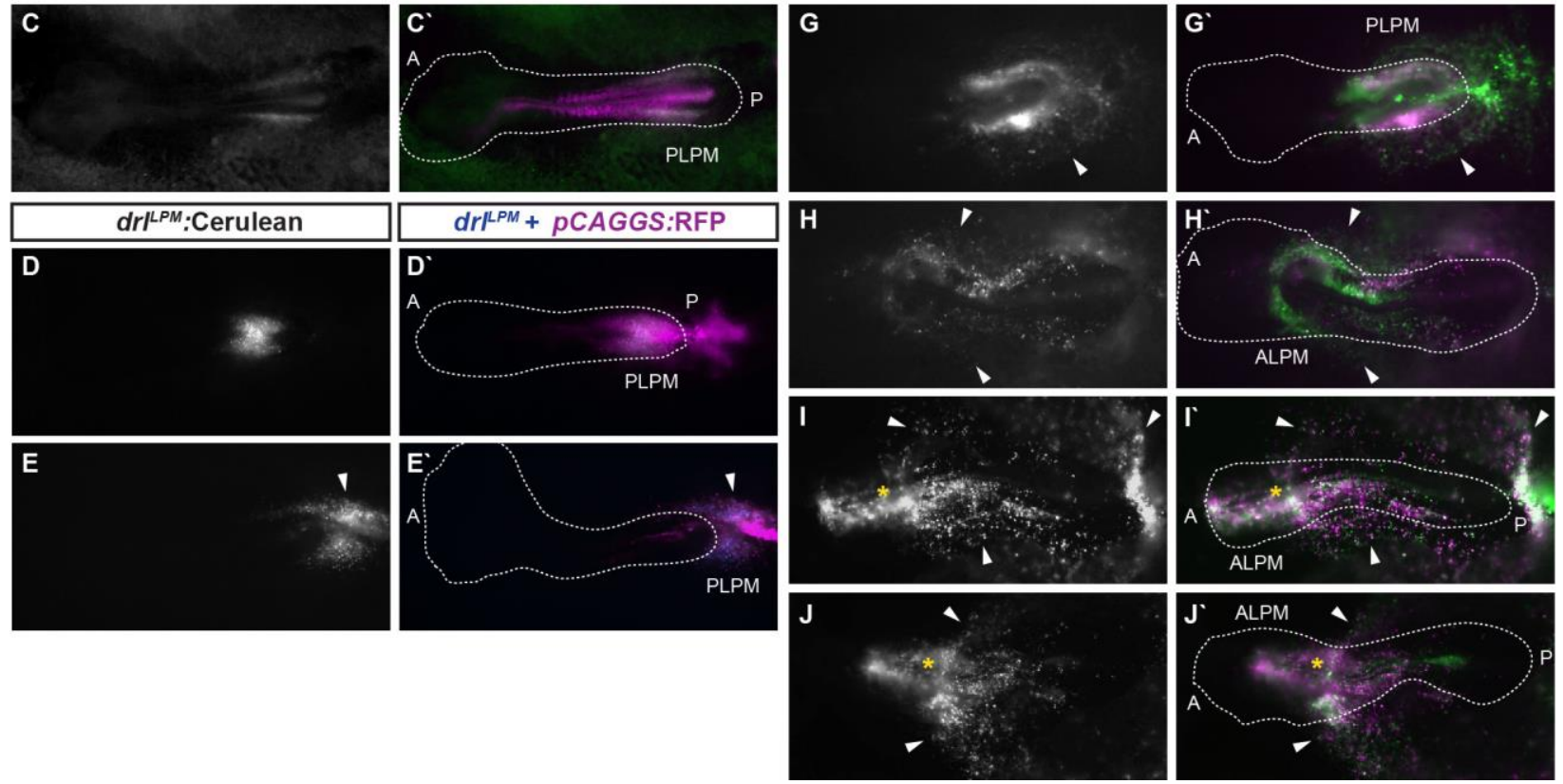

Fig. S5

Examples of electroporated chicken embryos with $\boldsymbol{d r l}^{L P M}$ reporters driving specific expression in the chicken LPM. (A) Brightfield picture of an HH9-staged chicken embryo with the zoomed-in region represented in a schematic, showing the different territories in the posterior. (B-J) Ex ovo cultured chicken embryos electroporated with $d r l^{L P M} c i s$-regulatory reporters as (B-C) $d r l^{L P M}: E G F P,(\mathbf{D}-\mathbf{E}) d r l^{L P M}:$ Cerulean $^{2}$, and (F-J) $d r l^{L P M}: m C h e r r y$ (magenta) together with control plasmid (B-E) $p C A G G S: R F P$ (magenta) or (F-J) $p C A G G S: E G F P$ at $\mathrm{HH} 3+/ \mathrm{HH} 4$. Arrowheads indicate extra-embryonic endothelial/blood progenitors and asterisks the heart field. The dash lines indicate the outline of the chicken embryos, with anterior (a) located to the left. 

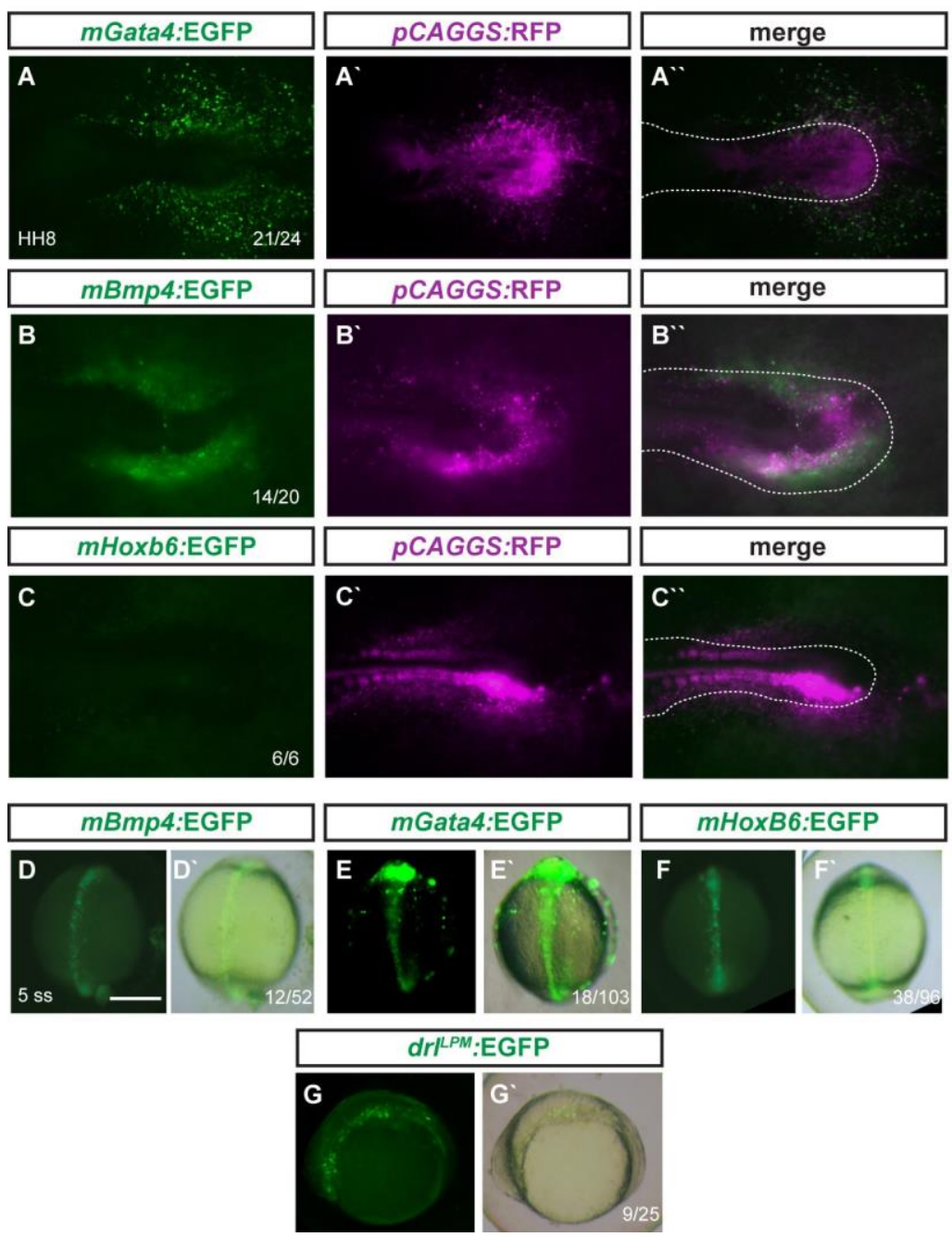

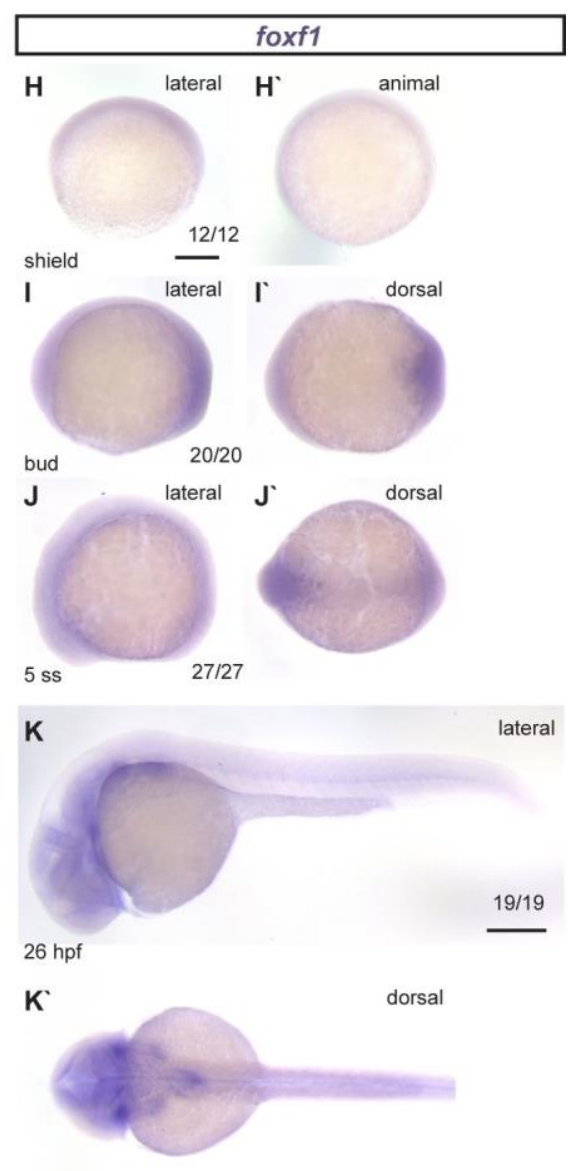

\section{Fig. S6}

No universal LPM-specific activity of previously characterized mouse LPM markers in both chicken and zebrafish. (A) The mouse Gata4, (B) Bmp4, and (c) Hoxb6 LPM elements as EGFP-expressing reporters electroporated together with control plasmid $p C A G G S: R F P$ (magenta) in ex ovo cultured chicken embryos at $\mathrm{HH} 3+/ \mathrm{H} 4$. Widefield imaging reveals expression in (A) extraembryonic blood vessels and (B) the posterior arch, or (C) unspecifically/absent. The dash lines indicate the outline of the chicken embryos. (D-G) Mouse Bmp4 (D), Gata4 (E), and Hoxb6 (F) LPM elements as EGFP-expressing Tol2-based reporter constructs injected in zebrafish embryos, showing confined axial mesoderm activity. (G) $d r l^{L P M}: E G F P$ as injection reference. (H-K) In situ hybridization (ISH) for foxfl from shield stage to $26 \mathrm{hpf}$ in zebrafish does not mark any LPM-derived structures or lineages. Scale bars in $(\mathbf{D}, \mathbf{H}, \mathbf{K}) 300 \mu \mathrm{m}$. 
bioRxiv preprint first posted online Feb. 7, 2018; doi: http://dx.doi.org/10.1101/261115. The copyright holder for this preprint (which was not peer-reviewed) is the author/funder, who has granted bioRxiv a license to display the preprint in perpetuity. It is made available under a CC-BY-NC-ND 4.0 International license.
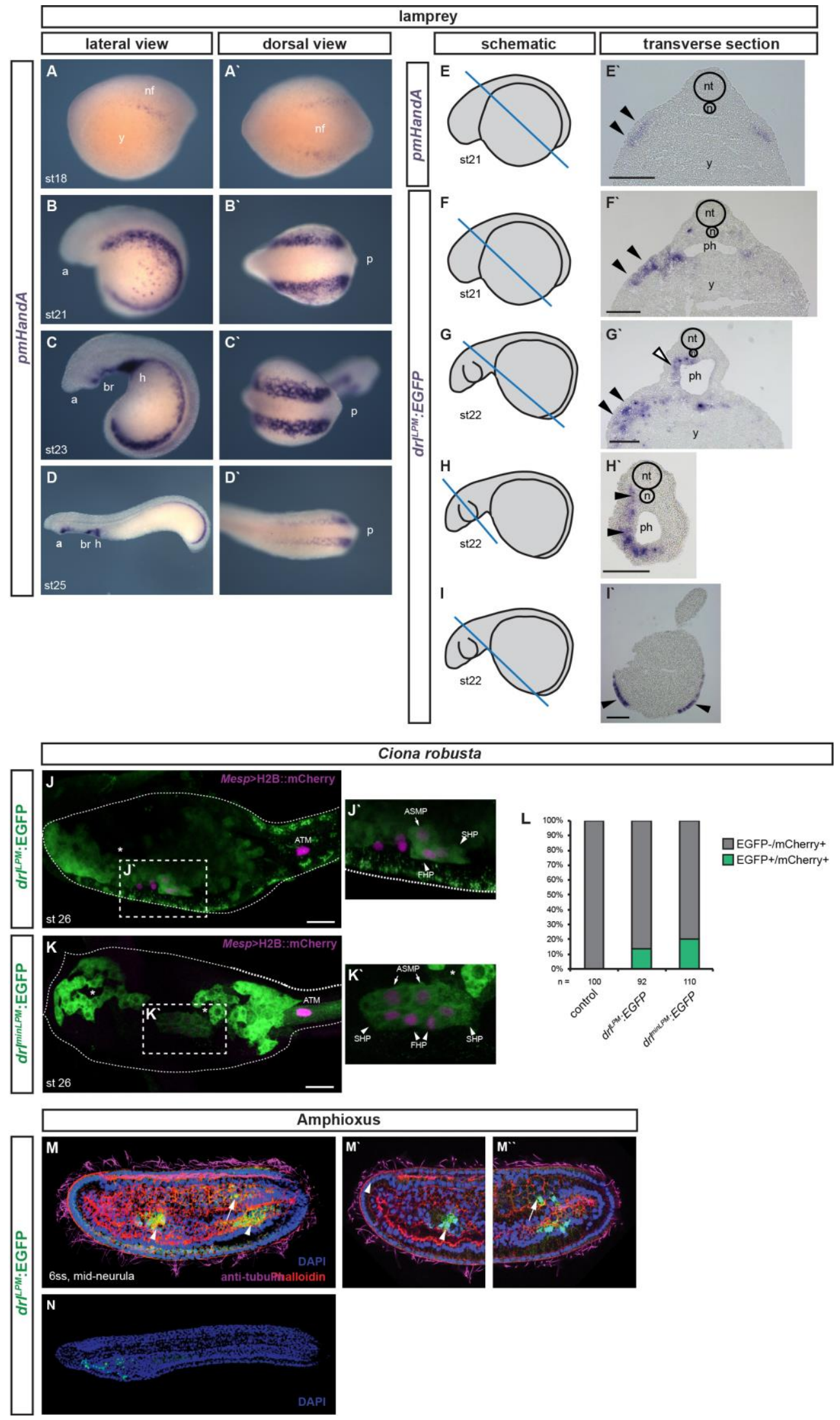


\section{Fig. S7}

$d r l^{L P M}$ drives specific reporter expression in lamprey, Ciona, and amphioxus. (A-D) Whole-mount ISH for pmHandA marks the LPM in lamprey embryos from neurula to hatching stages (st 18-25). (A) Expression is first detectable as bilateral stripes in the PLPM at st 18. (B,C) Later, the PLPM expression expands covering the yolk, and pmHandA is upregulated in the heart tube, branchial region, and caudal to the heart. Lateral and dorsal views are shown with the anterior (a) of the embryo to the left. (E-I) Transverse sections of fixed st 2122 embryos. The schematics indicate the plane of sectioning in blue and sections are shown with dorsal to the top. (E) ISH for pmHandA at st 21 marking the LPM (black arrowheads). (F-I) ISH for EGFP in transverse sections of $d r l^{L P M}: E G F P$ transient transgenic lamprey embryos fixed at st 21-22, showing enhancer activity in the anterior mesendoderm subjacent to the ectoderm ( $\mathbf{F}^{\mathbf{}}, \mathbf{G}^{\mathbf{}}$, black arrowheads), in the pharyngeal endoderm (G', white arrowhead), in the pharyngeal mesoderm (H', black arrowheads), and in some embryos in the ectoderm as commonly observed unspecific expression of reporter plasmids (I , black arrowheads). (J,K) Immunostaining for EGFP in Ciona larvae embryo (st 26) expressing $d r l$ reporters (green) and Mesp>H2B::mCherry to track the B7.5 nuclei cell lineage (red). Expression of $d r l^{L P M_{-}}(\mathbf{J})$ and $d r l^{\min L P M_{-}}(\mathbf{K})$ driven EGFP reporter in the larvae is stained in the mesenchymal lineage (white asterisks) and in the B7.5 cell progeny including ASM precursors (ASMP) (white arrows) and both cardiac first and second heart precursors (FHPs and SHPs) (white arrowheads), zoomed in ( $\left.\mathbf{J}, \mathbf{K}^{\prime}\right)$. Dashed line indicates midline. Anterior is to the left. (l) Proportion of larvae embryos expressing both GFP and mCherry in the B7.5 lineage when co-electroporated $d r l$ reporter and Mesp $>\mathrm{H} 2 \mathrm{~B}:: \mathrm{mCherry}$ in comparison to the control. N: number of electroporated larval halves. (M-M“) Confocal Z-stack of amphioxus embryo at mid-neurula stage (6 ss), injected with $d r l^{L P M}: E G F P$, showing specific reporter activity in the lateral endoderm (arrowhead), and ventral half of the somites (arrows). (N) At early larvae stage, the activity of $d r l^{L P M}$ reporter was observed in the endoderm of the pharynx $(\mathrm{n}=$ 15/30) and absent in the ventral mesoderm $(n=0 / 30)$. Embryos counterstained with Phalloidin (red), embryos/larvae with DAPI (blue), and anti-acetylated tubulin antibody (magenta). Lateral view shown as 3Drendering (M) or Z-stack sagittal sections (M',M“), anterior to the left and dorsal to the top. (N) At early larvae stage, the activity of $d r l^{L P M}$ reporter was observed in the endoderm of the pharynx $(\mathrm{n}=15 / 30)$ and absent in the ventral mesoderm $(n=0 / 30)$. Branchial region (br), heart $(h)$, neural folds $(n f)$, neural tube (nt), notochord (n), pharynx (ph), and yolk (y) labeled in (E-H). Scale bars in (E'-I') are $200 \mu \mathrm{m}$, and in (K) $25 \mu \mathrm{m}$. 

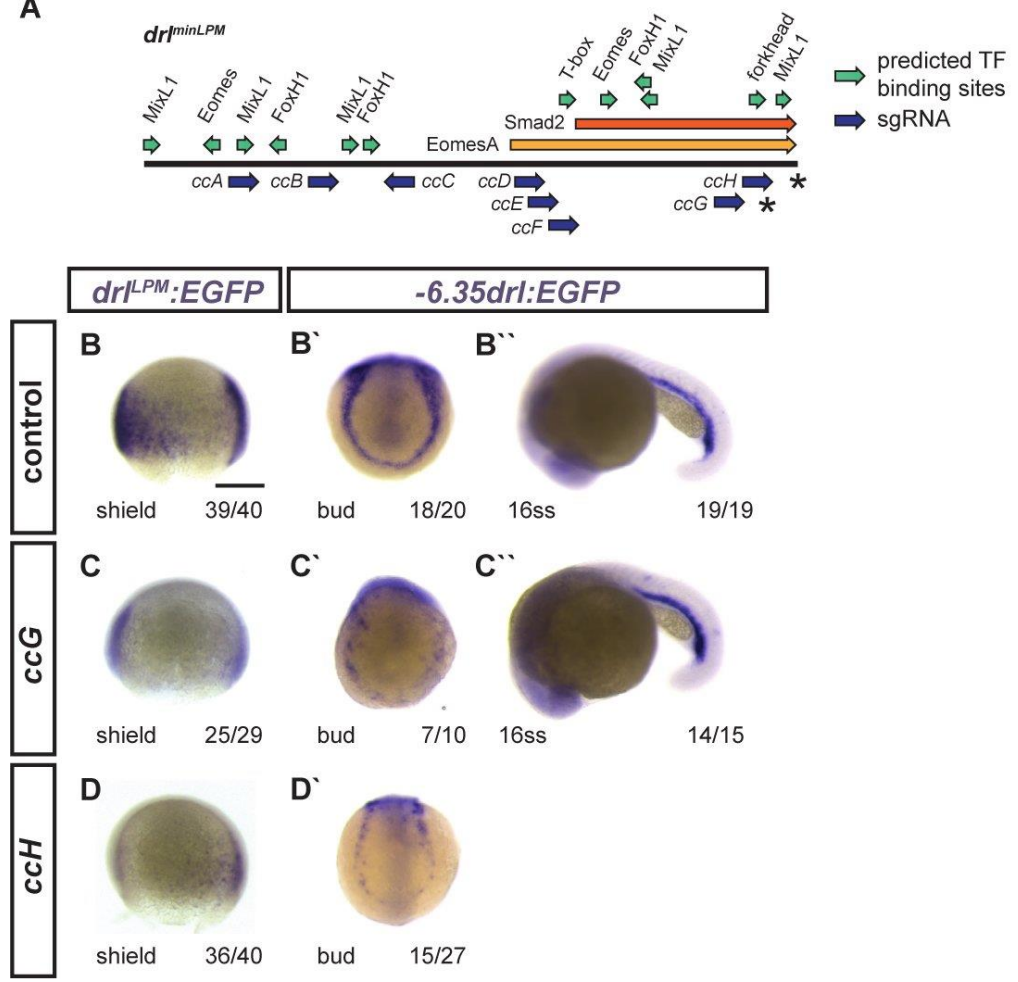

E

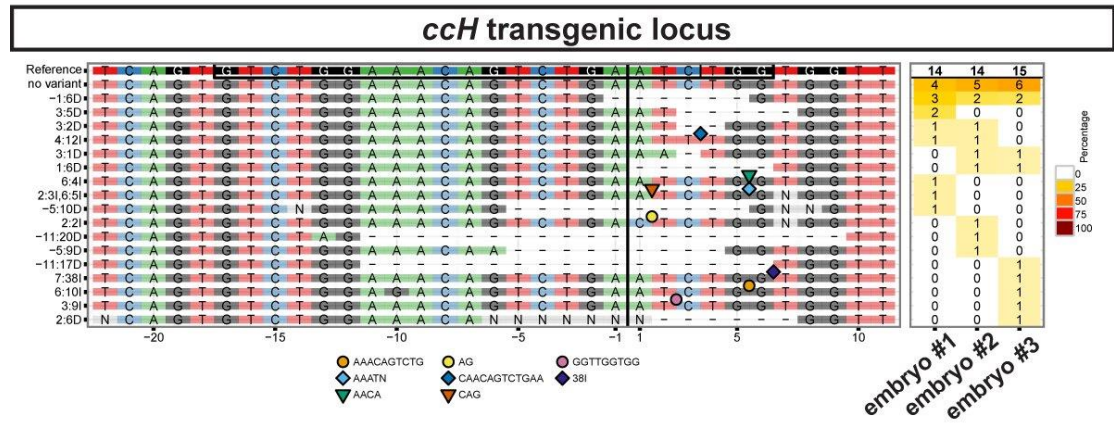

\section{Fig. S8}

Crispant analysis of the minimally required $\boldsymbol{d r} l^{\min L P M}$ region. (A) Schematic representation of the minimal $d r l^{\min L P M}$ region with transcription factor binding site predictions (JASPAR), Eomes and Smad2 ChIP-seq annotations, and individual sgRNAs annotated. (B-D) ISH for EGFP in $d r l^{L P M}: E G F P$ embryos individually mutagenized using reconstituted Cas9 protein-sgRNA complexes with either sgRNA (C) $c c G$ or $(\mathbf{D}) c c H$ (asterisks in $\mathbf{A}$ ), showing diminished reporter expression at bud stage compared to uninjected controls. $\left(\mathbf{B}^{\prime \prime}, \mathbf{C}^{\prime \prime}\right)$ Reporter expression remains functional at later stages, here shown for 16 ss. (E) Panel plot shows mutagenesis efficiency and allele spectrum in three representative $c c H$ crispants, as created by CrispRVariants. The genomic reference sequence is shown on top, with the $20 \mathrm{bp} c c H$ sgRNA followed by a 3 bp PAM indicated by the boxed regions. The Cas9 cleavage site is represented by a black vertical bar. Deletions are indicated by a '-', and inserted sequences by symbols. The right column of the plot shows the allele frequency, showing that some mutant alleles occurred in different crispants. Scale bar $250 \mu \mathrm{m}$. 
A Individual and combinatorial misexpression of VP16 / eng mRNA construct

\begin{tabular}{lll}
\hline & VP16 & eng \\
\hline EomesA & ++ & nc \\
\hline FoxH1 & nc & nc \\
\hline MixL1 & nc & nc \\
\hline Spr2 & nc & nc \\
\hline Osr1 & nc & nc \\
\hline ScI/Tal1 & nc & \\
\hline Mespaa & nc & \\
\hline Etv5a & -- & ++ \\
\hline Ntl & nc & \\
\hline E/F/M & +++ & nc \\
\hline
\end{tabular}

$++\quad d r l^{L P M}$ expression increase in native LPM territory

$+++d r l^{L P M}$ ectopic expression

-- $d r L^{L P M}$ expression decrease

nc no change in $d_{r l L P M}$ expression
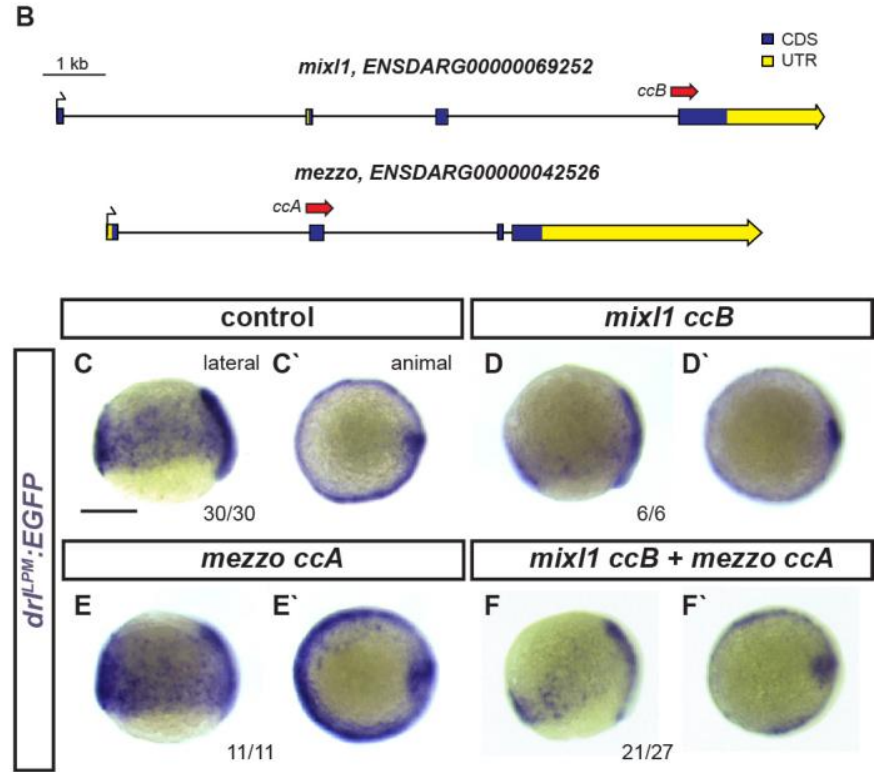

\section{Fig. S9}

mRNA-mediated misexpression screen uncovered Mixl1 as a third component acting on $\boldsymbol{d r}^{L P M}$. (A) Transcription factors were fused N-terminally with the transactivation domain VP16 or the engrailed (eng) repressor domain ORF, and tested for impact on $d r l^{L P M}$ reporter activity. (B) Schematic representation of the mixll and mezzo loci with the individual sgRNAs annotated. (C-F) Cas9 RNP-mediated crispant analysis by mRNA in situ hybridization of $E G F P$ expression in $d r l^{L P M}: E G F P$ embryos injected with (D) mixll $c c B,(\mathbf{E})$ mezzo $c c A$, and (F) mixll $c c B$ plus mezzo $c c A$ sgRNA; the resulting mosaic mixll $c c B$ crispants show diminished $d r l^{L P M}$ reporter expression at late gastrulation. Lateral and animal views are shown as indicated. Scale bar in (C) $250 \mu \mathrm{m}$. 


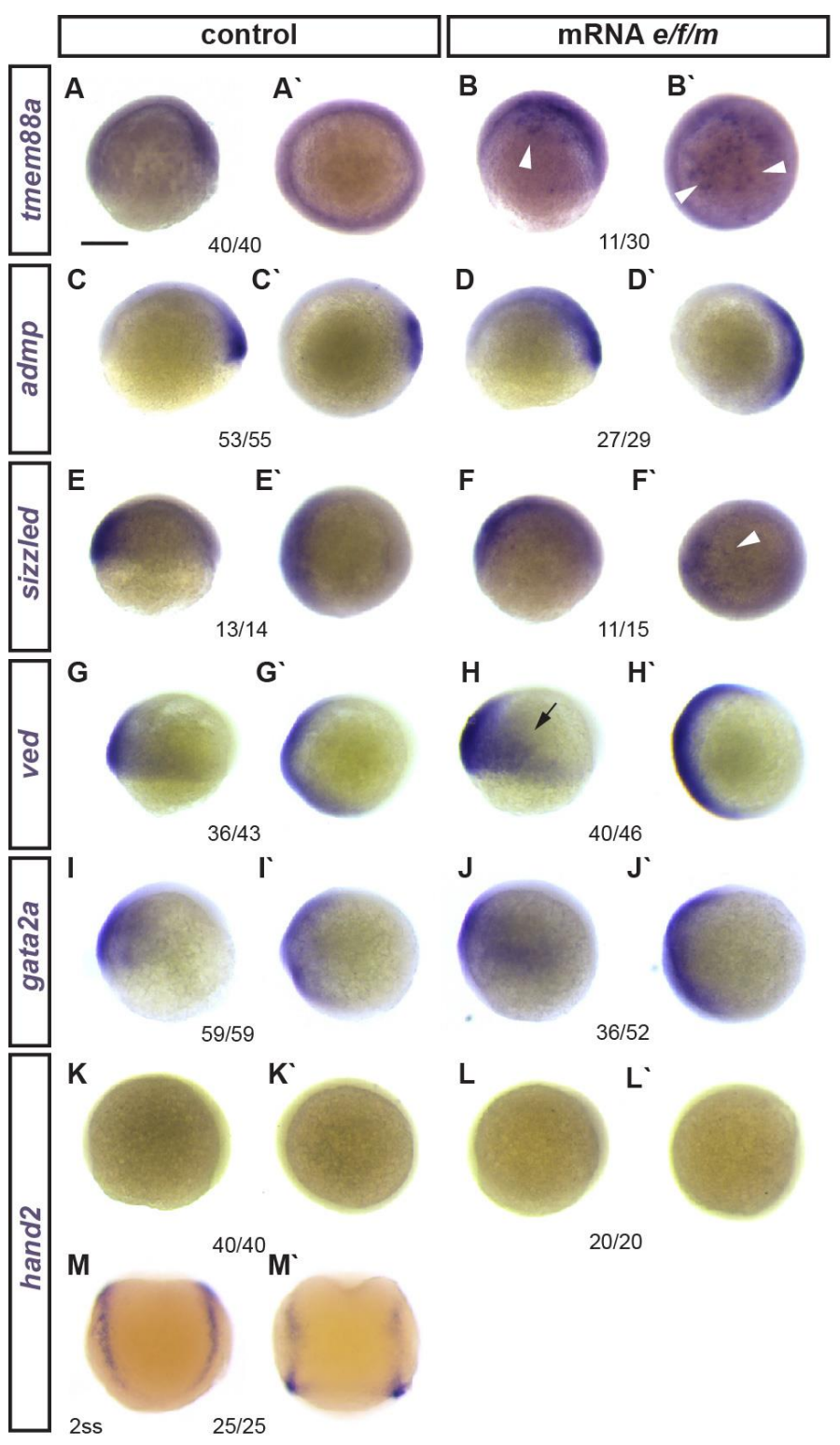

Fig. S10

$e / f / m$ misexpression drives ectopic expression of the LPM gene tmem 88 a while the expression domains of BMP target genes and the post-gastrulation LPM maker hand2 remain broadly intact. ISH for endogenous expression of tmem88a (ectopic expression, indicated by arrowheads) (A,B), admp (C,D), sizzled (slight increased expression, indicated by arrowhead) (E,F), ved (slight increased expression, indicated by arrow) (G,H), gata2a (I,j), and hand2 (K-M) in embryos injected with e/f/m mRNA compared to representative control embryos. Lateral and animal views are shown as indicated. Scale bar (A) $250 \mu \mathrm{m}$. 


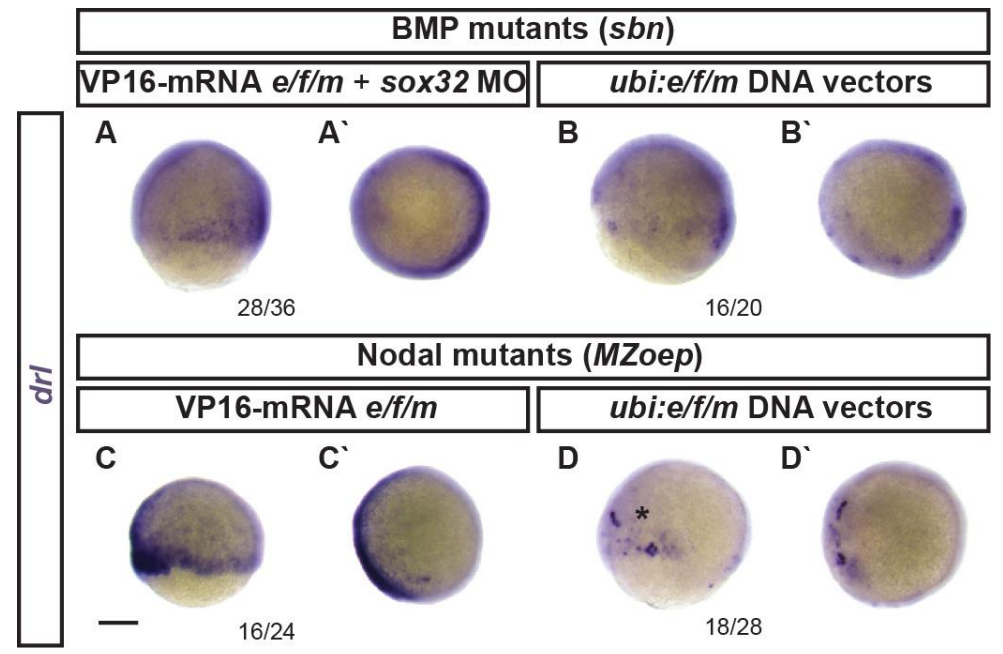

\section{Fig. S11}

VP16-e/f/m mRNA injections in Nodal and BMP mutants. (A,B) mRNA ISH for endogenous $d r l$ expression in BMP-mutant $(s b n)$ embryos injected with either (A) $V P 16-e / f / m m R N A$ in an endoderm-perturbed background (sox32 morpholino) or (B) ubi:e/f/m DNA vectors, showing enhanced ventral $d r l$ expression. (CD) Additional mRNA ISH for endogenous $d r l$ expression in Nodal-mutant (MZoep) embryos injected with (C) VP16-e/f/m mRNA or (D) $u b i: e / f / m$ DNA vectors. Scale bar in (A) $250 \mu \mathrm{m}$. 
Movie S1: 3D lightsheet imaging of the arising -6.35drl reporter-labeled LPM. SPIM time lapse imaging of zebrafish embryo transgenic for $-6.35 \mathrm{drl}$ :EGFP (green) together with nuclear marker $a c t b 2: \mathrm{H} 2 \mathrm{afva}-$ mCherry (magenta) to visualize LPM emergence from $50 \%$ epiboly to 10 ss.

Movie S2: Panoramic lightsheet imaging of the arising $\mathbf{- 6 . 3 5 d r l}$ reporter-labeled LPM. Mercator projection of SPIM time lapse imaging of zebrafish embryos transgenic for $-6.35 \mathrm{drl}$ :EGFP to visualize LPM emergence from $50 \%$ epiboly to 10 ss. From somitogenesis onwards, the embryo is oriented with anterior to the left and posterior to the right.

Movie S3: 3D lightsheet imaging showing overlap between -6.35drl reporter expression and sox17marked endoderm during gastrulation. SPIM time lapse imaging of $-6.35 \mathrm{drl}$ : $\mathrm{mCherry}$ (red) combined with sox 17:EGFP (green) to visualize the $d r l$-expressing emerging cell population versus endoderm from $50 \%$ epiboly until 16 ss.

Movie S4: Panoramic lightsheet imaging showing overlap between $\mathbf{- 6 . 3 5 d r l}$ reporter expression and sox 17-marked endoderm during gastrulation. Mercator projection of SPIM time lapse imaging of 6.35drl:mCherry (magenta) combined with sox17:EGFP to visualize the $d r l$-expressing emerging cell population versus endoderm from $50 \%$ epiboly until 16 ss. From somitogenesis onwards, the embryo is oriented with anterior to the left and posterior to the right. Double-positive cells during gastrulation and early somitogenesis are shown in blue. 